\title{
Exploring Cellular Interactions of Liposomes Using Protein Corona Fingerprints and Physicochemical Properties
}

\author{
Arafeh Bigdeli, ${ }^{\dagger}$ Sara Palchetti, ${ }^{\ddagger}$ Daniela Pozzi, ${ }^{\ddagger}$ Mohammad Reza Hormozi-Nezhad, ${ }^{\dagger}$ \\ Francesca Baldelli Bombelli, ${ }^{\S}$ Giulio Caracciolo, ${ }^{* \dagger}$ and Morteza Mahmoudi*,\#,\|l \\ ${ }^{\dagger}$ Department of Chemistry, Sharif University of Technology, Tehran 1113658639, Iran \\ ${ }^{\ddagger}$ Department of Molecular Medicine, “Sapienza” University of Rome, Rome 00185, Italy \\ $\S$ \\ Department of Chemistry, Materials and Chemical Engineering Giulio Natta, Politecnico di Milano, Milan 20133, Italy \\ Nanotechnology Research Center, Faculty of Pharmacy, Tehran University of Medical Sciences, Tehran 1316943551, Iran \\ "Stanford Cardiovascular Institute and Division of Cardiovascular Medicine, Stanford University School of Medicine, Stanford, \\ California 94305, United States
}

\begin{abstract}
To control liposomes fate and transport upon
contact with biofluids, it is essential to consider several parameters affecting the synthetic and biological identity of liposomes, as well as liposome-protein corona (PC) aspects. As a powerful tool in this data mining adventure, quantitative structure-activity relationship (QSAR) ap-proach is used to correlate physicochemical properties of liposomes and their PC fingerprints to multiple quantified biological responses. In the present study, the relationship between cellular interactions of a set of structurally diverse liposomal formulations and their physicochemical and PC properties has been investigated via linear and nonlinear QSAR models. Significant parameters affecting cellular uptake and cell viability of liposomes in two important cancer cell lines (PC3 and HeLa) have been identified. The developed QSARs have the capacity to be implemented in advanced targeted delivery of liposomal drugs.
\end{abstract}

KEYWORDS: liposomes, cellular uptake, cell viability, QSAR, protein corona, cancer

$\mathrm{L}$ iposomes are organic nanoscale vesicles that are clinically established as outstanding drug delivery systems, as well as diagnostic and treatment tools for several diseases, particularly cancer and fungal infections. ${ }^{1,2}$ Liposomes consist of single or multiple lipid bilayers encapsulating an aqueous compartment. Their unique selfassembled structure enables them to entrap desired hydrophilic/hydrophobic agents into their internal aqueous core/ phospholipid bilayer, making them versatile tools for drug delivery systems. Given their biocompatibility, biodegradability, ease of size, and surface tuning properties, liposomes have attracted much interest in nanomedicine in the last few decades and have found the potential to be further applied in theranostics. ${ }^{3}$

As with any other nanostructure upon entering the biological media, liposomes are also immediately surrounded by high levels of proteins (or other biomolecules), forming a rich protein shell, referred to as "protein corona" (PC). These evolving collection of proteins associated within the lipid surface control the surface properties of liposomes in the biological environment for a certain amount of time, determining what is "seen" by the living organism. ${ }^{4-6}$ Thus, the initial synthetic identity of the liposomes (i.e., size, shape, and surface chemistry) will turn into a new biological identity (i.e., size and aggregation state of the liposome, together with the structure and composition of the PC). The resulting biological identity may be far different from the synthetic identity of liposomes, effecting their fate and transport leading to unpredictable biological responses (e.g., biodistribution, uptake, opsonization, toxicity, kinetics).

Several factors such as size, shape, charge, surface chemistry, topography, and curvature of the liposome can regulate the formation of PC. There has been several recent studies investigating the formed PC around liposomes with different 
Table 1. Physicochemical Properties of Liposomal Formulations, Including Size of Bare Liposomes and Liposome-HP complexes, Zeta Potential of Bare Liposomes and Liposome-HP complexes, Micrograms of Proteins Bound to Liposomes after 1 h Incubation with HP

\begin{tabular}{|c|c|c|c|c|c|c|c|}
\hline & \multicolumn{2}{|c|}{ size $(n m)$} & \multicolumn{2}{|c|}{ PDI } & \multicolumn{2}{|c|}{ zeta potential $(\mathrm{mV})$} & \multirow{2}{*}{$\frac{\text { protein assay }(\mu \mathrm{g} / \mu \mathrm{L})}{+\mathrm{HP}}$} \\
\hline & $-\mathrm{HP}^{a}$ & $+\mathrm{HP}^{b}$ & $-\mathrm{HP}$ & $-\mathrm{HP}$ & $-\mathrm{HP}$ & $+\mathrm{HP}$ & \\
\hline $\mathrm{L} 1$ & $97 \pm 4$ & $235 \pm 37$ & $0.12 \pm 0.01$ & $0.21 \pm 0.04$ & $52 \pm 2.1$ & $-20.7 \pm 0.6$ & $7.29 \pm 1.12$ \\
\hline $\mathrm{L} 2$ & $112 \pm 11$ & $228 \pm 4$ & $0.17 \pm 0.01$ & $0.23 \pm 0.02$ & $48.5 \pm 0.9$ & $-17.9 \pm 0.7$ & $9.73 \pm 1.10$ \\
\hline L3 & $135 \pm 8$ & $259 \pm 21$ & $0.14 \pm 0.04$ & $0.12 \pm 0.04$ & $30.1 \pm 3.4$ & $-19.3 \pm 1.8$ & $5.06 \pm 0.45$ \\
\hline L4 & $128 \pm 17$ & $150 \pm 6$ & $0.11 \pm 0.02$ & $0.15 \pm 0.01$ & $47.4 \pm 0.9$ & $-23.0 \pm 1.1$ & $10.55 \pm 0.89$ \\
\hline L5 & $131 \pm 7$ & $182 \pm 13$ & $0.10 \pm 0.01$ & $0.16 \pm 0.05$ & $26.1 \pm 2.9$ & $-25.5 \pm 1.4$ & $5.51 \pm 0.54$ \\
\hline L6 & $157 \pm 10$ & $211 \pm 14$ & $0.17 \pm 0.01$ & $0.23 \pm 0.01$ & $58.2 \pm 4.3$ & $-28.5 \pm 2.3$ & $6.70 \pm 0.62$ \\
\hline L7 & $108 \pm 4$ & $216 \pm 2$ & $0.14 \pm 0.02$ & $0.22 \pm 0.04$ & $34.5 \pm 5.5$ & $-17.9 \pm 2.3$ & $4.02 \pm 0.43$ \\
\hline L8 & $125 \pm 10$ & $207 \pm 12$ & $0.18 \pm 0.02$ & $0.21 \pm 0.02$ & $46.2 \pm 2.2$ & $-20.4 \pm 4.5$ & $5.47 \pm 0.56$ \\
\hline L9 & $255 \pm 23$ & $329 \pm 12$ & $0.12 \pm 0.02$ & $0.15 \pm 0.01$ & $33.1 \pm 2.4$ & $-15.4 \pm 1.2$ & $4.36 \pm 0.28$ \\
\hline L10 & $193 \pm 15$ & $236 \pm 18$ & $0.13 \pm 0.02$ & $0.19 \pm 0.03$ & $42.1 \pm 1.2$ & $-19.3 \pm 1.4$ & $3.93 \pm 0.27$ \\
\hline L11 & $191 \pm 23$ & $301 \pm 18$ & $0.11 \pm 0.02$ & $0.15 \pm 0.05$ & $-8.4 \pm 1.9$ & $-22.1 \pm 2.3$ & $2.94 \pm 0.32$ \\
\hline L12 & $136 \pm 1$ & $152 \pm 12$ & $0.10 \pm 0.03$ & $0.15 \pm 0.02$ & $-26.6 \pm 0.2$ & $-18.2 \pm 0.8$ & $8.50 \pm 1.09$ \\
\hline L13 & $126 \pm 23$ & $171 \pm 11$ & $0.11 \pm 0.03$ & $0.13 \pm 0.02$ & $-14.2 \pm 1.1$ & $-28.6 \pm 1.4$ & $4.06 \pm 0.51$ \\
\hline $\mathrm{L} 14$ & $144 \pm 4$ & $178 \pm 6$ & $0.09 \pm 0.01$ & $0.14 \pm 0.01$ & $30.2 \pm 2.9$ & $-29.3 \pm 3.5$ & $4.91 \pm 0.64$ \\
\hline $\mathrm{L} 15$ & $114 \pm 1$ & $137 \pm 6$ & $0.08 \pm 0.01$ & $0.16 \pm 0.03$ & $18 \pm 3.3$ & $-13.9 \pm 0.6$ & $2.70 \pm 0.21$ \\
\hline L16 & $123 \pm 6$ & $169 \pm 6$ & $0.12 \pm 0.03$ & $0.17 \pm 0.03$ & $17.9 \pm 1.2$ & $-24.5 \pm 1.2$ & $1.50 \pm 0.03$ \\
\hline $\mathrm{L} 17$ & $103 \pm 10$ & $115 \pm 1$ & $0.09 \pm 0.03$ & $0.13 \pm 0.04$ & $9.9 \pm 2.2$ & $-13.2 \pm 0.9$ & $1.10 \pm 0.12$ \\
\hline
\end{tabular}

${ }^{a}$ Bare liposomes. ${ }^{b}$ Liposome-HP complexes.

synthetic identities. ${ }^{8-11}$ For instance, liposomes with charged surfaces tend to adsorb more proteins than those with neutral surfaces. Accordingly, liposomes with anionic or cationic phospholipid head groups activate a complement more efficiently than those bearing neutral head groups. In addition to the physicochemical properties of the liposome itself, proteins source and concentration, incubation time and temperature are also included among environmental factors shaping the liposome-PC. ${ }^{12-15}$ For instance, it has been shown that liposomes incubated in mouse plasma are more negatively charged than those in human plasma and bear PCs less enriched in opsonins and plentiful in apolipoproteins. This implies a dissimilar circulation time and pharmacokinetic profile of liposomes in the bloodstream of mice, compared to humans. ${ }^{16}$

Quantitative mapping of the relationships between liposomes' synthetic identity, biological identity and biological response enables researchers to thoroughly understand and control their nano-bio interactions in biofluids, which are essential for future developments in this research area. However, developing these correlations will require extensive studies, high-throughput techniques, and new strategies. As a promising approach in this regard, quantitative structureactivity relationship (QSAR) allows to predict the biological impact of PC formation on liposomes bioresponse and facilitates the identification of more meaningful relationships between the synthetic/biological identity of liposomes and their biological outcome. Recently, Chan et al. ${ }^{17}$ established a quantitative database of PC characterization and used serum PC fingerprints along with nanoparticle properties to predict the cell association of a library of gold nanoparticles with diverse sizes and surface coatings. According to the presented framework, in another study, Cohen et al. ${ }^{18}$ developed linear and nonlinear QSAR models for predicting nano-bio interactions of the previously introduced library of inorganic nanoparticles. However, Pozzi et al. ${ }^{19}$ have demonstrated that efficient predictive modeling of nanoparticle behavior in vivo requires accurate knowledge of PC fingerprints in circulating biological media, rather than static incubation, which is used as a model for protein adsorption so far. Thus, in order to take into account the effect of PC formation in QSAR studies, the $\mathrm{PC}$ composition formed under suitable dynamic conditions is likely the most accurate descriptor to be considered in this regard.

Moving toward liposomes as representative organic nanoparticles, a QSAR approach has been proposed herein to show how PC fingerprints and physicochemical properties of liposomes can predict their cellular interactions. Cellular uptake and cell viability were chosen as the most common biological profiles, revealing the physiological behavior of liposomes in different application of interest. Unlike the predetermined reports, in this study we probed several cellular responses to the corona coated liposomes, leading to deeper understanding of the biological behavior of these nanospecies. Moreover, the effect of protein corona formation has been carefully considered by not only introducing protein corona fingerprints within the descriptor set, but also measuring the cellular responses with and without precoating the liposomes with human plasma proteins.

\section{RESULTS AND DISCUSSION}

A set of 17 liposomal formulations was synthesized, followed by characterization steps before and after incubation in Human Plasma (HP). The prepared formulations differed in their lipid composition and were characterized by different size and surface charge (see Methods for details). The formulations included anionic, cationic, and neutral-charged liposomes with an averaged hydrodynamic size ranging from about 100 to $250 \mathrm{~nm}$, creating a library of physicochemical properties. Hydro-dynamic sizes and zeta potential measurements of bare liposomes and liposome-HP complexes, together with protein assay results (micrograms of proteins bound to liposomes after $1 \mathrm{~h}$ incubation with $\mathrm{HP}$ ), are provided in Table 1 . Interaction with pure HP was generally found to promote a significant increase in the hydrodynamic size (ranging between 12 and $138 \mathrm{~nm}$ ) and the polydispersity index (PDI). Following interaction 
Table 2. Descriptors Used in QSAR Models Including LPP and PCF

Liposome Physicochemical Properties (LPP)

$-\mathrm{HP}$

Size $(\mathrm{nm})$

Molecular Weight $(\mathrm{g} / \mathrm{mol})$

Surface area per liposome $\left(\mathrm{cm}^{2}\right)$

Mol (per liposome)

Zeta Potential $(\mathrm{mV})$

Protein Corona Fingerprints (PCF) abbrev.

HPX

YWHAZ

SERPINC1

PLTP

$\mathrm{CFH}$

ILK

ANGPTL6

$\mathrm{CP}$

COL18A1

MMRN1

C1R

IGLC2

IGHG1

KRT1

APOC1

IGHG2

APOB

TF

KRT2

ACTB

KRT10

C4B

IGHK8

A2M

HPR

IGHM

ORM1

HP

KRT5

KRT14

PON1

IGHA1

RBP4

C1QB

APOA5

SHBG

ORM2

KRT16

APOC4

APOF

F9

IGHC8

C1QC

A1BG

F10

KRT6A

DCD

AGLL5

TUBB1

SAA4

TUBA4A

FN1 \begin{tabular}{l}
\multicolumn{1}{c}{$+\mathrm{HP}$} \\
Size $(\mathrm{nm})$ \\
Zeta potential $(\mathrm{mV})$ \\
Protein Density $(\mu \mathrm{g} / \mu \mathrm{L})$
\end{tabular}

full name

Hemopexin

14-3-3 protein zeta/delta

Antithrombin-III

Phospholipid transfer protein

Complement factor $\mathrm{H}$

Integrin-linked protein kinase

Angiopoietin-related protein 6

Ceruloplasmin

Collagen alpha-1(XVIII) chain

Multimerin-1

Complement $\mathrm{C} 1 \mathrm{r}$ subcomponent

Ig lambda-2 chain $\mathrm{C}$ regions

Ig gamma-1 chain $\mathrm{C}$ region

Keratin, type II cytoskeletal 1

Apolipoprotein C-I

Ig gamma-2 chain $\mathrm{C}$ region

Apolipoprotein B-100

Serotransferrin

Keratin, type II cytoskeletal 2 epidermal

Actin, cytoplasmic 1

Keratin, type I cytoskeletal 10

Complement C4-B

Ig kappa chain V-III region $\mathrm{HAH}$

Alpha-2-macroglobulin

Haptoglobin-related protein

Ig mu chain $\mathrm{C}$ region

Alpha-1-acid glycoprotein 1

Haptoglobin

Keratin, type II cytoskeletal 5

Keratin, type I cytoskeletal 14

Serum paraoxonase/arylesterase 1

Ig alpha-1 chain $\mathrm{C}$ region

Retinol-binding protein 4

Complement C1q subcomponent subunit B

Apolipoprotein A-V

Sex hormone-binding globulin

Alpha-1-acid glycoprotein 2

Keratin, type I cytoskeletal 16

Apolipoprotein C-IV

Apolipoprotein F

Coagulation factor IX

Ig heavy chain $\mathrm{V}-\mathrm{III}$ region $\mathrm{VH} 26$

Complement $\mathrm{C} 1 \mathrm{q}$ subcomponent subunit $\mathrm{C}$

Alpha-1B-glycoprotein

Coagulation factor $\mathrm{X}$

Keratin, type II cytoskeletal 6A

Dermcidin

Immunoglobulin lambda-like polypeptide 5

Tubulin beta-1 chain

Serum amyloid A-4 protein

Tubulin alpha-4A chain

Fibronectin 
Table 2. continued

\begin{tabular}{|c|c|c|c|}
\hline \multicolumn{4}{|c|}{ Protein Corona Fingerprints (PCF) } \\
\hline abbrev. & full name & abbrev. & full name \\
\hline $\mathrm{APOH}$ & Beta-2-glycoprotein 1 & COMP & Cartilage oligomeric matrix protein \\
\hline THBS1 & Thrombospondin-1 & SERPINA4 & Kallistatin \\
\hline ITGB3 & Integrin beta-3 & GP1BA & Platelet glycoprotein $\mathrm{Ib}$ alpha chain \\
\hline KRT9 & Keratin, type I cytoskeletal 9 & IGHC8 & Ig heavy chain V-III \\
\hline AHSG & Alpha-2-HS-glycoprotein & TUBA1B & Tubulin alpha-1B chain \\
\hline LPA & Apolipoprotein(a) & FBLN1 & Fibulin-1 \\
\hline FLNA & Filamin-A & THBS4 & Thrombospondin-4 \\
\hline IGJ & Immunoglobulin $\mathrm{J}$ chain & AFM & Afamin \\
\hline $\mathrm{C} 5$ & Complement C5 & IGHL8 & Ig lambda chain V-III region LO \\
\hline AGT & Angiotensinogen & F5 & Coagulation factor $\mathrm{V}$ \\
\hline GC & Vitamin D-binding protein & VWF & von Willebrand factor \\
\hline SEPP1 & Selenoprotein $\mathrm{P}$ & SLC4A1 & Band 3 anion transport protein \\
\hline COLEC10 & Collectin-10 & SERPINF1 & Pigment epithelium-derived factor \\
\hline APOD & Apolipoprotein D & CALR & Calreticulin \\
\hline SERPINF2 & Alpha-2-antiplasmin & CFHR5 & Complement factor H-related protein 5 \\
\hline TPM4 & Tropomyosin alpha-4 chain & LCAT & Phosphatidylcholine-sterol acyltransferase \\
\hline C8G & Complement component C8 gamma chain & $\mathrm{CF} 1$ & Complement factor I \\
\hline APOL1 & Apolipoprotein L1 & CPN2 & Carboxypeptidase $\mathrm{N}$ subunit 2 \\
\hline VCL & Vinculin & HSP90B1 & Endoplasmin \\
\hline PGLYRP2 & $\mathrm{N}$-acetylmuramoyl-L-alanine amidase & CNDP1 & Beta-Ala-His dipeptidase \\
\hline $\mathrm{C} 7$ & Complement component $\mathrm{C} 7$ & HRNR & Hornerin \\
\hline $\mathrm{C} 8 \mathrm{~B}$ & Complement component $\mathrm{C} 8$ beta chain & PRG4 & Proteoglycan 4 \\
\hline C6 & Complement component $\mathrm{C} 6$ & FLG2 & Filaggrin-2 \\
\hline SERPING1 & Plasma protease $\mathrm{C} 1$ inhibitor & TUBB & Tubulin beta chain \\
\hline $\mathrm{C} 8 \mathrm{~A}$ & Complement component $\mathrm{C} 8$ alpha chain & COLEC11 & Collectin-11 \\
\hline C1QA & Complement $\mathrm{C} 1 \mathrm{q}$ subcomponent subunit $\mathrm{A}$ & IGHG3 & Ig gamma-3 chain $\mathrm{C}$ region \\
\hline GP9 & Platelet glycoprotein IX & IGHG8 & Ig heavy chain $\mathrm{V}-\mathrm{III}$ region $\mathrm{TRO}$ \\
\hline FERMT3 & Fermitin family homologue 3 & $\mathrm{CD} 14$ & Monocyte differentiation antigen CD14 \\
\hline PLG & Plasminogen & LGALS3BP & Galectin-3-binding protein \\
\hline CETP & Cholesteryl ester transfer protein & LYZ & Lysozyme C \\
\hline SERPINA3 & Alpha-1-antichymotrypsin & IGBUT & Ig heavy chain $\mathrm{V}-\mathrm{III}$ region $\mathrm{BUT}$ \\
\hline ITIH4 & Interalpha-trypsin inhibitor heavy chain $\mathrm{H} 4$ & SAA1 & Serum amyloid A-1 protein \\
\hline VTN & Vitronectin & HPX & Hemopexin \\
\hline FGB & Fibrinogen beta chain & YWHAZ & $14-3-3$ protein zeta/delta \\
\hline F2 & Prothrombin & SERPINC1 & Antithrombin-III \\
\hline ALB & Serum albumin & PLTP & Phospholipid transfer protein \\
\hline APOA2 & Apolipoprotein A-II & $\mathrm{CFH}$ & Complement factor $\mathrm{H}$ \\
\hline FGG & Fibrinogen gamma chain & ILK & Integrin-linked protein kinase \\
\hline IGKC & Ig kappa chain $\mathrm{C}$ region & ANGPTL6 & Angiopoietin-related protein 6 \\
\hline $\mathrm{C} 3$ & Complement C3 & $\mathrm{CP}$ & Ceruloplasmin \\
\hline FGA & Fibrinogen alpha chain & COL18A1 & Collagen alpha-1(XVIII) chain \\
\hline APOC2 & Apolipoprotein C-II & MMRN1 & Multimerin-1 \\
\hline Clusterin & Clusterin & $\mathrm{C} 1 \mathrm{R}$ & Complement $\mathrm{C} 1 \mathrm{r}$ subcomponent \\
\hline HABP2 & Hyaluronan-binding protein 2 & IGLC2 & Ig lambda-2 chain $\mathrm{C}$ regions \\
\hline APOC3 & Apolipoprotein C-III & IGHG1 & Ig gamma- 1 chain $\mathrm{C}$ region \\
\hline PROS1 & Vitamin K-dependent protein $S$ & KRT1 & Keratin, type II cytoskeletal 1 \\
\hline C4BPA & C4b-binding protein alpha chain & APOC1 & Apolipoprotein C-I \\
\hline TTR & Transthyretin & IGHG2 & Ig gamma-2 chain $\mathrm{C}$ region \\
\hline $\mathrm{HBB}$ & Hemoglobin subunit beta & APOB & Apolipoprotein B-100 \\
\hline AMBP & Protein AMBP & $\mathrm{TF}$ & Serotransferrin \\
\hline HBA1 & Hemoglobin subunit alpha & KRT2 & Keratin, type II cytoskeletal 2 epidermal \\
\hline ITIH2 & Interalpha-trypsin inhibitor heavy chain $\mathrm{H} 2$ & АСТВ & Actin, cytoplasmic 1 \\
\hline ITIH1 & Interalpha-trypsin inhibitor heavy chain $\mathrm{H} 1$ & KRT10 & Keratin, type I cytoskeletal 10 \\
\hline ITIH3 & Interalpha-trypsin inhibitor heavy chain $\mathrm{H} 3$ & $\mathrm{C} 4 \mathrm{~B}$ & Complement C4-B \\
\hline PROC & Vitamin K-dependent protein C & IGHK8 & Ig kappa chain $\mathrm{V}-\mathrm{III}$ region $\mathrm{HAH}$ \\
\hline PROZ & Vitamin K-dependent protein Z & $\mathrm{A} 2 \mathrm{M}$ & Alpha-2-macroglobulin \\
\hline LUM & Lumican & HPR & Haptoglobin-related protein \\
\hline APOA1 & Apolipoprotein A-I & IGHM & Ig mu chain $\mathrm{C}$ region \\
\hline KNG1 & Kininogen-1 & ORM1 & Alpha-1-acid glycoprotein 1 \\
\hline
\end{tabular}


Table 2. continued

\begin{tabular}{|c|c|c|c|}
\hline abbrev. & full name & abbrev. & full name \\
\hline SERPIND1 & Heparin cofactor 2 & HP & Haptoglobin \\
\hline APOE & Apolipoprotein E & KRT5 & Keratin, type II cytoskeletal 5 \\
\hline C1S & Complement $\mathrm{C}$ 1s subcomponent & KRT14 & Keratin, type I cytoskeletal 14 \\
\hline SERPINA1 & Alpha-1-antitrypsin & PON1 & Serum paraoxonase/arylesterase 1 \\
\hline SERPINA10 & Protein Z-dependent protease inhibitor & IGHA1 & Ig alpha-1 chain $\mathrm{C}$ region \\
\hline С4BРB & C4b-binding protein beta chain & RBP4 & Retinol-binding protein 4 \\
\hline GSN & Gelsolin & $\mathrm{C} 1 \mathrm{QB}$ & Complement $\mathrm{C} 1 \mathrm{q}$ subcomponent subunit $\mathrm{B}$ \\
\hline MYL6 & Myosin light polypeptide 6 & APOAS & Apolipoprotein A-V \\
\hline RAP1B & Ras-related protein Rap- $1 \mathrm{~b}$ & SHBG & Sex hormone-binding globulin \\
\hline LBP & Lipopolysaccharide-binding protein & ORM2 & Alpha-1-acid glycoprotein 2 \\
\hline C9 & Complement component C9 & KRT16 & Keratin, type I cytoskeletal 16 \\
\hline SPP2 & Secreted phosphoprotein 24 & APOC4 & Apolipoprotein $\mathrm{C}-\mathrm{IV}$ \\
\hline TLN1 & Talin-1 & APOF & Apolipoprotein $\mathrm{F}$ \\
\hline CFB & Complement factor B & F9 & Coagulation factor IX \\
\hline ITGA2B & Integrin alpha-IIb & IGHC8 & Ig heavy chain V-III region $\mathrm{VH} 26$ \\
\hline STOM & Erythrocyte band 7 integral membrane protein & C1QC & Complement $\mathrm{C} 1 \mathrm{q}$ subcomponent subunit $\mathrm{C}$ \\
\hline MYH9 & Myosin-9 & AlBG & Alpha-1B-glycoprotein \\
\hline APOM & Apolipoprotein M & F10 & Coagulation factor $\mathrm{X}$ \\
\hline MASP1 & Mannan-binding lectin serine protease 1 & KRT6A & Keratin, type II cytoskeletal 6A \\
\hline IgKC5 & Ig kappa chain V-IV region Len & DCD & Dermcidin \\
\hline GAPDH & Glyceraldehyde-3-phosphate dehydrogenase & AGLL5 & Immunoglobulin lambda-like polypeptide 5 \\
\hline HRG & Histidine-rich glycoprotein & TUBB1 & Tubulin beta-1 chain \\
\hline APOA4 & Apolipoprotein A-IV & SAA4 & Serum amyloid A-4 protein \\
\hline CRP & C-reactive protein & TUBA4A & Tubulin alpha-4A chain \\
\hline PFN1 & Profilin-1 & FN1 & Fibronectin \\
\hline $\mathrm{APOH}$ & Beta-2-glycoprotein 1 & COMP & Cartilage oligomeric matrix protein \\
\hline THBS1 & Thrombospondin-1 & SERPINA4 & Kallistatin \\
\hline ITGB3 & Integrin beta- 3 & GP1BA & Platelet glycoprotein $\mathrm{Ib}$ alpha chain \\
\hline KRT9 & Keratin, type I cytoskeletal 9 & IGHC8 & Ig heavy chain V-III \\
\hline AHSG & Alpha-2-HS-glycoprotein & TUBA1B & Tubulin alpha-1B chain \\
\hline LPA & Apolipoprotein(a) & FBLN1 & Fibulin-1 \\
\hline FLNA & Filamin-A & THBS4 & Thrombospondin-4 \\
\hline IGJ & Immunoglobulin $\mathrm{J}$ chain & AFM & Afamin \\
\hline $\mathrm{C} 5$ & Complement C5 & IGHL8 & Ig lambda chain V-III region LO \\
\hline AGT & Angiotensinogen & F5 & Coagulation factor $\mathrm{V}$ \\
\hline GC & Vitamin D-binding protein & VWF & von Willebrand factor \\
\hline SEPP1 & Selenoprotein $\mathrm{P}$ & SLC4A1 & Band 3 anion transport protein \\
\hline COLEC10 & Collectin-10 & SERPINF1 & Pigment epithelium-derived factor \\
\hline APOD & Apolipoprotein D & CALR & Calreticulin \\
\hline SERPINF2 & Alpha-2-antiplasmin & CFHR5 & Complement factor $\mathrm{H}$-related protein 5 \\
\hline TPM4 & Tropomyosin alpha-4 chain & LCAT & Phosphatidylcholine-sterol acyltransferase \\
\hline C8G & Complement component C8 gamma chain & CF1 & Complement factor I \\
\hline APOL1 & Apolipoprotein L1 & $\mathrm{CPN} 2$ & Carboxypeptidase $\mathrm{N}$ subunit 2 \\
\hline VCL & Vinculin & HSP90B1 & Endoplasmin \\
\hline PGLYRP2 & $\mathrm{N}$-acetylmuramoyl-L-alanine amidase & CNDP1 & Beta-Ala-His dipeptidase \\
\hline C7 & Complement component $\mathrm{C} 7$ & HRNR & Hornerin \\
\hline $\mathrm{C} 8 \mathrm{~B}$ & Complement component $\mathrm{C} 8$ beta chain & PRG4 & Proteoglycan 4 \\
\hline C6 & Complement component C6 & FLG2 & Filaggrin-2 \\
\hline SERPING1 & Plasma protease $\mathrm{C} 1$ inhibitor & TUBB & Tubulin beta chain \\
\hline $\mathrm{C} 8 \mathrm{~A}$ & Complement component C8 alpha chain & COLEC11 & Collectin-11 \\
\hline $\mathrm{C} 1 \mathrm{QA}$ & Complement $\mathrm{C} 1 \mathrm{q}$ subcomponent subunit $\mathrm{A}$ & IGHG3 & Ig gamma-3 chain $\mathrm{C}$ region \\
\hline GP9 & Platelet glycoprotein IX & IGHG8 & Ig heavy chain $\mathrm{V}$-III region TRO \\
\hline FERMT3 & Fermitin family homologue 3 & $\mathrm{CD} 14$ & Monocyte differentiation antigen CD14 \\
\hline PLG & Plasminogen & LGALS3BP & Galectin-3-binding protein \\
\hline CETP & Cholesteryl ester transfer protein & LYZ & Lysozyme C \\
\hline SERPINA3 & Alpha-1-antichymotrypsin & IGBUT & Ig heavy chain $\mathrm{V}-\mathrm{III}$ region $\mathrm{BUT}$ \\
\hline ITIH4 & Interalpha-trypsin inhibitor heavy chain $\mathrm{H} 4$ & SAA1 & Serum amyloid A-1 protein \\
\hline VTN & Vitronectin & HPX & Hemopexin \\
\hline FGB & Fibrinogen beta chain & YWHAZ & $14-3-3$ protein zeta/delta \\
\hline
\end{tabular}


Table 2. continued

\begin{tabular}{llll}
\multicolumn{1}{c}{ full name } & \multicolumn{1}{c}{ Protein Corona Fingerprints (PCF) } & \multicolumn{1}{c}{ full name } \\
\multicolumn{1}{rl}{ abbrev. } & & \multicolumn{1}{c}{ abbrev. } & Antithrombin-III \\
F2 & Prothrombin & SERPINC1 & Phospholipid transfer protein \\
ALB & Serum albumin & PLTP & Complement factor H \\
APOA2 & Apolipoprotein A-II & CFH & Integrin-linked protein kinase \\
FGG & Fibrinogen gamma chain & ILK & Angiopoietin-related protein 6 \\
IGKC & Ig kappa chain C region & ANGPTL6 & Ceruloplasmin \\
C3 & Complement C3 & CP & Collagen alpha-1(XVIII) chain \\
FGA & Fibrinogen alpha chain & COL18A1 & Multimerin-1 \\
APOC2 & Apolipoprotein C-II & MMRN1 & Complement C1r subcomponent \\
Clusterin & Clusterin & C1R & Ig lambda-2 chain C regions \\
HABP2 & Hyaluronan-binding protein 2 & IGLC2 & Ig gamma-1 chain C region \\
APOC3 & Apolipoprotein C-III & IGHG1 & Keratin, type II cytoskeletal 1 \\
PROS1 & Vitamin K-dependent protein S & KRT1 & Apolipoprotein C-I \\
C4BPA & C4b-binding protein alpha chain & APOC1 & Ig gamma-2 chain C region
\end{tabular}
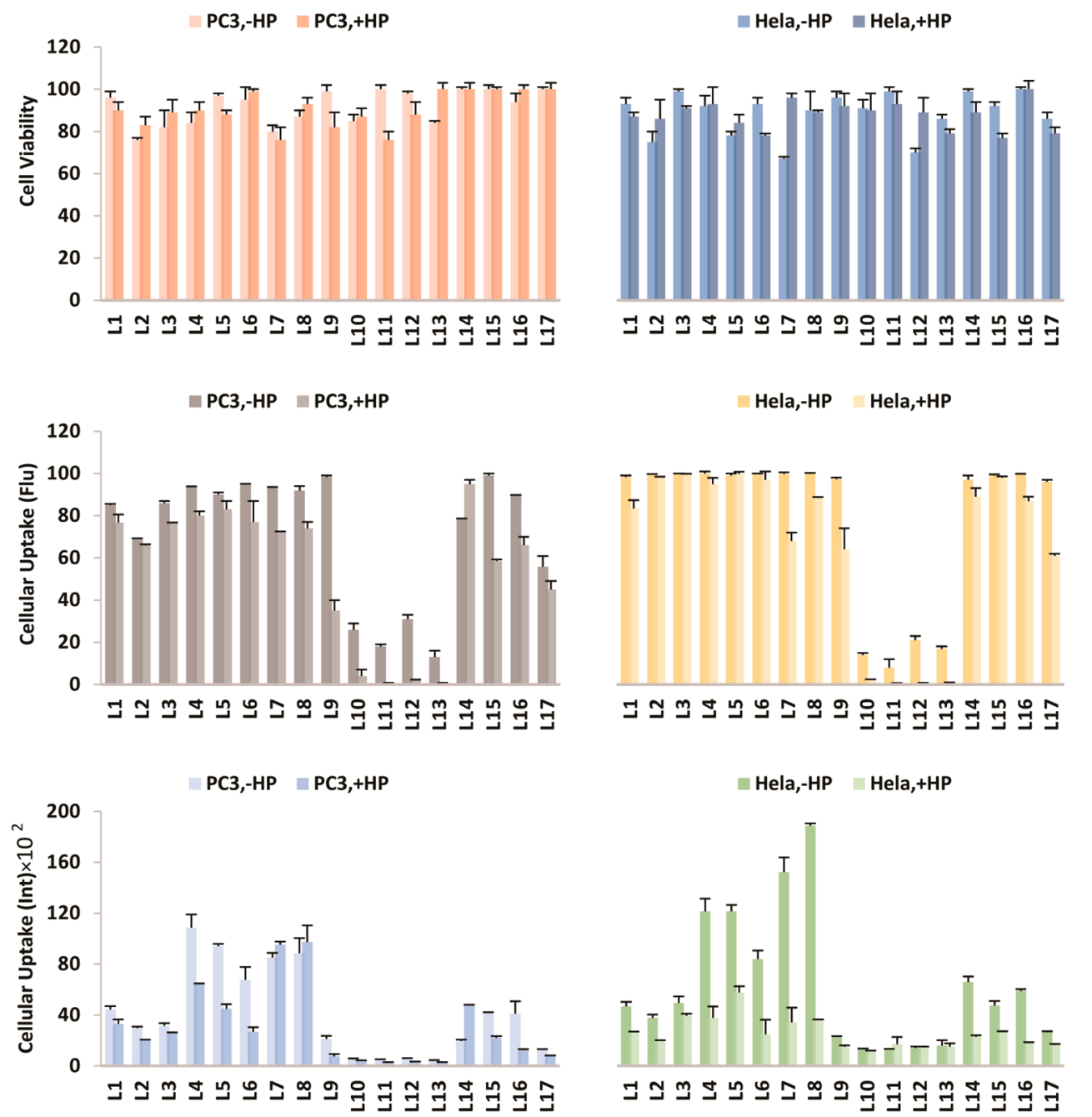

Figure 1. Cell viability, percentage of fluorescent cells, and mean intensity in PC3 and HeLa cell lines of bare liposomes (-HP) and liposome-HP complexes (+HP).

with $\mathrm{HP}$, zeta potential of cationic liposomes reversed to negative values, while it remained negative for anionic ones (L11-L13). In particular, incubation of the liposomes in HP led to a "normalization" of the zeta potential to an average value of about $-21.04 \pm 4.98 \mathrm{mV}$ independently of the pristine surface charge. This behavior was already observed for metal
NPs incubated in HP indicating that likely the main driving force in the protein-NP interaction is not electrostatic. ${ }^{20-23}$ After incubation with $\mathrm{HP}$ for $1 \mathrm{~h}$ at $37{ }^{\circ} \mathrm{C}$, HP-liposome complexes were isolated from excess of plasma and an average of $5.20 \pm 0.64 \mu \mathrm{g} / \mathrm{mL}$ adsorbed proteins was quantified per each liposomal formulation. In particular, it can be seen that 

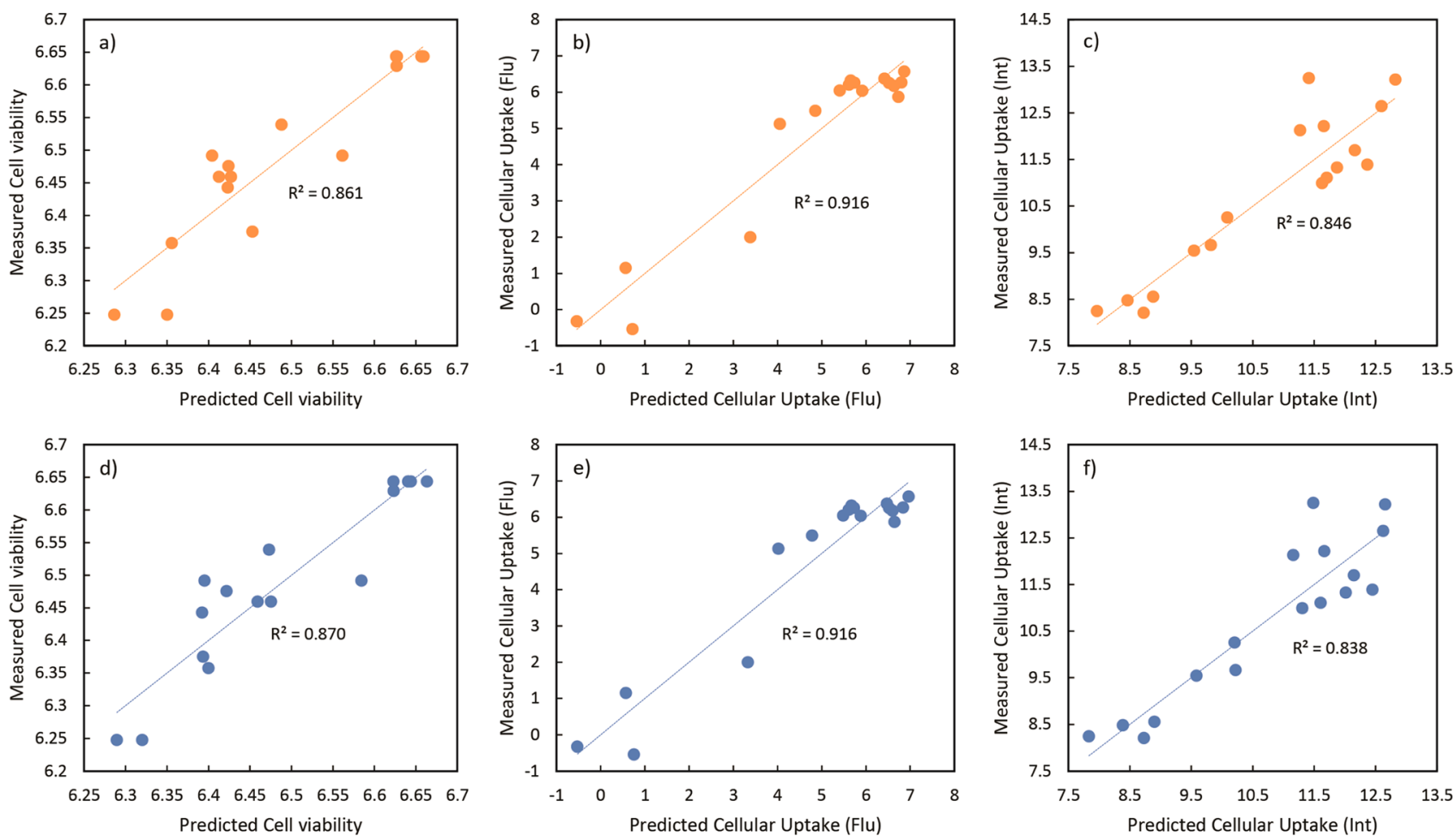

Figure 2. Measured bioresponse values (log2-transformed) of PC3 cells after administration of liposome-HP complexes versus their predicted values by linear MLR (top) and nonlinear ANN (bottom) QSARs. (a and d) Cell viability; (b and e) cellular uptake_Fluorescence; (c and f) cellular uptake_Intensity. The data for HeLa cell line and -HP/+HP is depicted in Figures S1-S3.

higher amount of adsorbed protein was associated with highly positive lipid surface, while a lower protein enrichment was found for slightly positive lipid surfaces that were partially coated by polyethyleneglygol (PEG) chains. The characterization information on the liposomes, before and after incubation in $\mathrm{HP}$, referred to as "liposome physicochemical properties (LPP)", was used as the first descriptor set, including 23 LPPs (briefly listed in Table 2).

The PC composition of each liposomal formulation was quantitatively characterized by liquid chromatography tandem mass spectrometry (LC-MS/MS). The results of the obtained $\mathrm{PC}$ compositions are presented in Table S1 (i.e., descriptors 1633) of the Supporting Information. For formulation, a quantitative profile of $633 \mathrm{HP}$ proteins was provided. The relative abundance of the adsorbed proteins was named "PC fingerprints (PCF)" and it represents the second descriptor set (Table 2). The PCF obtained for the liposome formulations confirmed that the physicochemical properties of liposomes can dictate the composition of the PC. ${ }^{6,8}$ It also showed that the PC composition is not necessarily enriched with the most abundant proteins in plasma. ${ }^{8,24}$

Twelve different biological end points were measured for each liposomal formulation, including their toxicity and cellular uptake, either with or without incubation with HP and in two

different cancer cell lines. The corresponding data do not show any global trend, emphasizing the necessity of utilizing a comprehensive modeling approach for a thorough investiga-tion. For instance, it can be seen in Figure 1 that the overall percentage of fluorescent cells is significantly lower for L10L13 in both cell lines and this reduction is enhanced after incubation in HP. We can notice that those formulations are all formed with negative charged liposomes, but the charge does not seem to be the only parameter influencing the uptake as also L10 (positive charged) shows a modest uptake. Thus, the phenomenon has to be due to the interplay between PC, size and charge variations. In fact, this hypothesis could not justify the low uptake value for L10, bearing a positive surface charge, implying that probably more variables other than solely charge might be responsible in this particular bioresponse. In addition, PC formation (together with its effects on surface charge of the particles) has shown to significantly affect the biological response of certain liposome formulation (e.g., cellular uptake intensity values for HeLa cells has dramatically decreased after HP incubation for L7-L8), while in some other cases, none or very little changes upon corona formation were observed (e.g., cell viability in PC3 cells in for L14-L17). These examples confirmed that for an in-depth and systematic investigation on the impact of PC on liposomes biological behavior, it is necessary to make some step further and implement it with QSAR analysis, rather than only considering the effect of one variable at a time. Moreover, it is necessary to take into account both descriptor sets: LPPs (revealing synthetic and biological identities of liposomes) and PCFs (covering the PC information) for a comprehensive analysis.

To develop linear and nonlinear correlations between the determined descriptors $(x)$ and the measured biological end points $(y)$, multiple linear regressions (MLR) and artificial neural networks (ANN) were accomplished to model $y$ as a function of $x, y=f(x)$. Figure 2 compares the performance of the constructed linear and nonlinear regression models for predicting the three different biological response profiles of the liposome data set $(\mathrm{L} 1-\mathrm{L} 17)$. The results for the remaining nine response profiles can be found in the Supporting Information in Figure $S 1-S 3$. The $R^{2}$ values displayed in Figure 2 were obtained including all liposome formulations in the model that was created using the best-selected descriptors chosen during 
Table 3. Summary of Linear and Nonlinear QSAR Models for All Bioresponses

\begin{tabular}{|c|c|c|c|c|c|c|c|c|c|c|c|c|c|}
\hline & & \multicolumn{4}{|c|}{ cell viability } & \multicolumn{4}{|c|}{ cellular uptake (flu) } & \multicolumn{4}{|c|}{ cellular uptake (int) } \\
\hline & & PC3 & PC3 & HeLa & $\mathrm{HeLa}$ & PC3 & PC3 & HeLa & $\mathrm{HeLa}$ & PC3 & PC3 & HeLa & HeLa \\
\hline & & $-\mathrm{HP}$ & $+\mathrm{HP}$ & $-\mathrm{HP}$ & $+\mathrm{HP}$ & $-\mathrm{HP}$ & $+\mathrm{HP}$ & $-\mathrm{HP}$ & $+\mathrm{HP}$ & $-\mathrm{HP}$ & $+\mathrm{HP}$ & $-\mathrm{HP}$ & $+\mathrm{HP}$ \\
\hline \multirow[t]{5}{*}{ Linear MLR model } & $R_{\text {Fit }}^{2}$ & 0.79 & 0.88 & 0.82 & 0.71 & 0.93 & 0.94 & 0.91 & 0.93 & 0.88 & 0.89 & 0.84 & 0.86 \\
\hline & $R_{\mathrm{cv}}^{2}$ & 0.59 & 0.65 & 0.55 & 0.55 & 0.64 & 0.66 & 0.69 & 0.65 & 0.60 & 0.60 & 0.57 & 0.56 \\
\hline & RMSE $_{\text {Fit }}$ & 0.06 & 0.04 & 0.07 & 0.05 & 0.23 & 0.57 & 0.34 & 0.71 & 0.52 & 0.53 & 0.48 & 0.23 \\
\hline & $\mathrm{RMSE}_{\mathrm{cv}}$ & 0.20 & 0.16 & 0.26 & 0.18 & 1.07 & 3.42 & 1.95 & 3.91 & 2.02 & 1.89 & 1.55 & 0.79 \\
\hline & $R_{\mathrm{y}-\text { rand }}^{2}$ & 0.12 & 0.14 & 0.19 & 0.14 & 0.20 & 0.14 & 0.23 & 0.21 & 0.17 & 0.13 & 0.16 & 0.14 \\
\hline \multirow[t]{5}{*}{ Nonlinear ANN model } & $R_{\mathrm{Fit}}^{2}$ & 0.64 & 0.84 & 0.77 & 0.72 & 0.97 & 0.99 & 0.98 & 0.99 & 0.83 & 0.77 & 0.76 & 0.82 \\
\hline & $R_{\mathrm{cv}}^{2}$ & 0.53 & 0.58 & 0.49 & 0.65 & 0.69 & 0.74 & 0.67 & 0.73 & 0.57 & 0.54 & 0.52 & 0.58 \\
\hline & RMSE $_{\text {Fit }}$ & 0.06 & 0.04 & 0.06 & 0.08 & 0.09 & 0.19 & 0.10 & 0.15 & 0.48 & 0.62 & 0.52 & 0.23 \\
\hline & $\operatorname{RMSE}_{\mathrm{cv}}$ & 0.17 & 0.13 & 0.23 & 0.13 & 0.76 & 1.94 & 1.09 & 2.00 & 1.58 & 1.63 & 1.35 & 0.64 \\
\hline & $R_{y-\text { rand }}^{2}$ & 0.09 & 0.16 & 0.14 & 0.13 & 0.11 & 0.12 & 0.15 & 0.16 & 0.11 & 0.15 & 0.12 & 0.10 \\
\hline
\end{tabular}
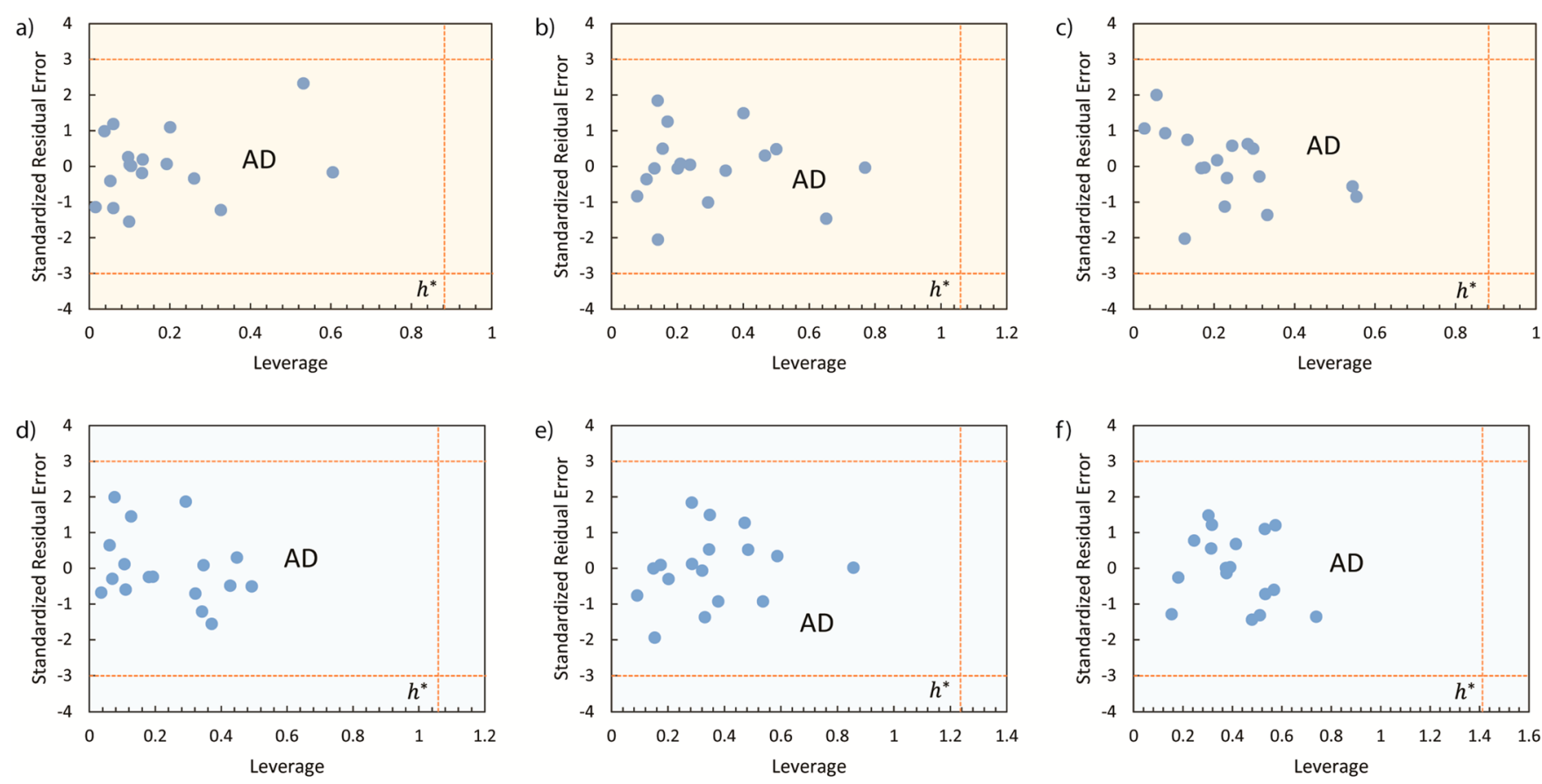

Figure 3. Applicability domain of the linear and nonlinear QSAR models identified by William's plot. (a) MLR, cell viability; (b) MLR, cellular uptake_Flu; (c) MLR, cellular uptake_Int; (d) ANN, cell viability; (e) ANN, cellular uptake_Flu; (f) ANN, cellular uptake_Int. The data shown here is for PC $3,+\mathrm{HP}$. The AD for HeLa cell line and $-\mathrm{HP} /+\mathrm{HP}$ can be found in Figures S4-S6.

the variable selection step. The accuracy of the developed QSARs was further assessed via Monte Carlo cross validation, in which the data set was repeatedly partitioned into random training and prediction sets. The former was used to build the model and also to choose the most suitable variables, while the latter was used to evaluate the prediction ability of the created model. The training and prediction accuracies were quantified by the average coefficient of determination for the training and prediction sets, referred to as fitting $\left(R_{\text {fit }}^{2}\right)$ and cross-validation $\left(R_{\text {cv }}^{2}\right)$, respectively (Table 3$)$. Both linear and nonlinear models showed high accuracy, deduced by their acceptable $R_{\text {fit }}^{2}$ and $R_{\mathrm{cv}}^{2}$ values. The results revealed that ANN models provided a slightly better correlation for the fluorescent cellular uptake of liposomes compared to the MLR models. This disclosed the nonlinear nature of the corresponding biointeraction. In contrast, for the other two bioresponses, the MLR models seemed to perform better in correlating the liposomes properties to their physiological end points. Determining the linear or nonlinear behavior beyond a specific biological interaction can lead to superior design of the liposomes. Another index, the root-mean-square error (RMSE), was also measured for the training and prediction sets for evaluating the predictability of the developed models. Accordingly, the low $\mathrm{RMSE}_{\text {fit }}$ and $\mathrm{RMSE}_{\mathrm{cy}}$ values also confirmed the satisfactory accuracy of the developed models. The linear and nonlinear QSARs demonstrated to have a wide range of applicability as they predict the right behavior of all liposome formulations except for L14 in its linear correlation to cellular uptake in HeLa cells (Figures 3 and S4-S6).

The selected variables in each model are summarized in Table 4. The first point that can be concluded is that the best variables selected in all QSAR models included both LPP and PCF descriptors, implying the importance of the information arising from both physicochemical properties and liposome- PC. This was further tested by building the QSAR models using only LPP descriptors (Table 5). Comparing the results between this table and Table 3 confirms the role of information-rich PCF descriptors that contribute to better QSAR models. The 
Table 4. List of Selected Variables in Linear and Nonlinear QSAR Models for Each Bioresponse

\begin{tabular}{|c|c|c|c|c|c|c|c|c|c|}
\hline & descripto & & 1 st & 2nd & $3 \mathrm{rd}$ & 4th & 5th & 6th & 7th \\
\hline \multirow[t]{8}{*}{ Cell Viability } & PC3, -HP & MLR & DCD & SA & $\mathrm{CP}$ & RBP4 & & & \\
\hline & & ANN & $\mathrm{CP}$ & C9 & Size, change & SA & Tot $\operatorname{Pr}$ & & \\
\hline & $\mathrm{PC} 3,+\mathrm{HP}$ & MLR & SAA4 & LPA & COL18A1 & Size, change & & & \\
\hline & & ANN & SAA4 & LPA & COL18A1 & $\mathrm{CP}$ & & & \\
\hline & HeLa, -HP & MLR & IGHL8 & Size, change & $\mathrm{CP}$ & $\mathrm{ZP}$, change & & & \\
\hline & & ANN & IGHL8 & Size, change & $\mathrm{CP}$ & $\mathrm{ZP}$, change & CETP & & \\
\hline & HeLa, +HP & MLR & KRT5 & IGLC2 & $\mathrm{ZP},+\mathrm{HP}$ & & & & \\
\hline & & ANN & SAA4 & IGLC2 & IGHL8 & $\mathrm{ZP},+\mathrm{HP}$ & KRT5 & & \\
\hline \multirow[t]{8}{*}{ Cellular Uptake (Flu) } & PC3, -HP & MLR & $\operatorname{IgKC5}$ & Size, $-\mathrm{HP}$ & APOF & GSN & PRG4 & & \\
\hline & & ANN & KRT6A & $\mathrm{ZP},+\mathrm{HP}$ & APOF & HPX & GSN & $\mathrm{C} 8 \mathrm{~B}$ & \\
\hline & PC3, +HP & MLR & Size,-HP & AHSG & $\mathrm{ZP},-\mathrm{HP}$ & APOF & & & \\
\hline & & ANN & PRG4 & $\mathrm{ZP},-\mathrm{HP}$ & Size, change & $\mathrm{ZP},+\mathrm{HP}$ & & & \\
\hline & HeLa, -HP & MLR & HPX & LPA & APOF & & & & \\
\hline & & ANN & PRG4 & Size, $-\mathrm{HP}$ & AHSG & HPX & SHBG & APOF & \\
\hline & $\mathrm{HeLa},+\mathrm{HP}$ & MLR & PRG4 & APOF & AHSG & $\mathrm{ZP},-\mathrm{HP}$ & & & \\
\hline & & ANN & $\mathrm{ZP},-\mathrm{HP}$ & AHSG & SHBG & APOF & PRG4 & & \\
\hline \multirow[t]{8}{*}{ Cellular Uptake (Int) } & PC3, -HP & MLR & $\mathrm{ZP}$, change & APOF & Size, $-\mathrm{HP}$ & $\mathrm{ZP},-\mathrm{HP}$ & & & \\
\hline & & ANN & AGT & $\mathrm{C} 1 \mathrm{R}$ & $\mathrm{ZP},-\mathrm{HP}$ & GSN & F9 & APOF & $\mathrm{ZP},+\mathrm{HP}$ \\
\hline & PC3, +HP & MLR & APOF & PROC & Size, $-\mathrm{HP}$ & $\mathrm{ZP},-\mathrm{HP}$ & & & \\
\hline & & ANN & Size, $-\mathrm{HP}$ & $\mathrm{ZP}$, change & PROC & $\mathrm{ZP},-\mathrm{HP}$ & APOF & & \\
\hline & HeLa, -HP & MLR & $\mathrm{ZP}$, change & LYZ & $\mathrm{ZP},-\mathrm{HP}$ & & & & \\
\hline & & ANN & COMP & APOF & Size, $-\mathrm{HP}$ & PROC & & & \\
\hline & HeLa, +HP & MLR & TUBB1 & HPR & SA & $\mathrm{ZP},-\mathrm{HP}$ & & & \\
\hline & & ANN & HPR & TUBB1 & SERPING1 & SA & $\mathrm{ZP},+\mathrm{HP}$ & $\mathrm{ZP},-\mathrm{HP}$ & \\
\hline
\end{tabular}

Table 5. Summary of Linear and Nonlinear QSAR Models When Considering Only LPPs as Descriptors

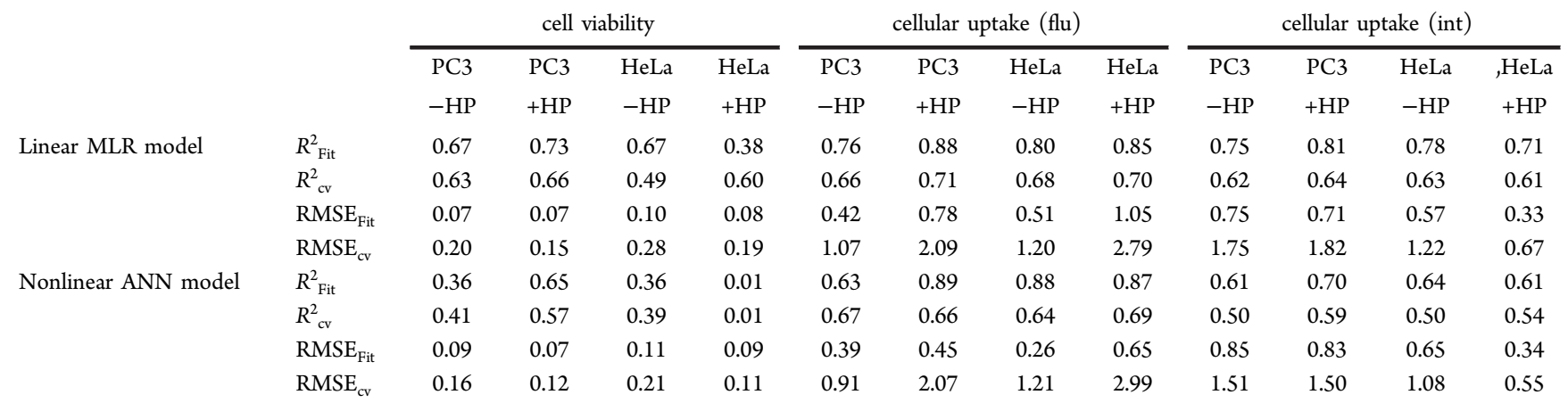
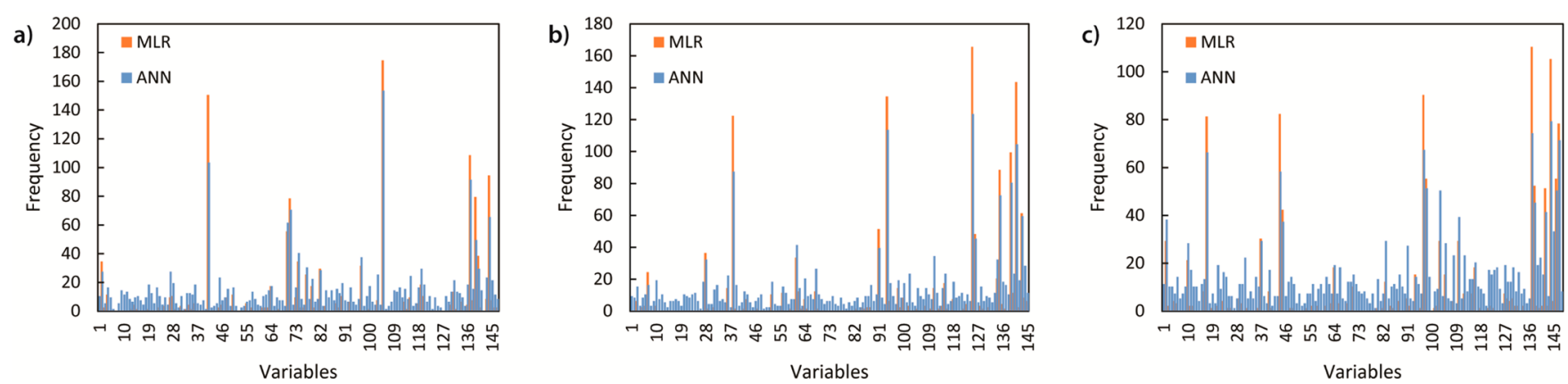

Figure 4. Frequency plot of variables in linear (orange) and nonlinear (blue) QSAR models. The higher frequency of a variable indicates it has been more selected during the variable selection iterations. (a) Cell viability, (b) cellular uptake_Flu, (c) cellular uptake_Int. The frequency plots in this figure are related to PC3 cell line and +HP. The results for HeLa cells and -HP/+HP can be found in Figures S7-S9.

descriptors listed for each model provide a comprehensive comparison framework for the important parameters in each biological response. For example, it seems that the IGHL8, IGLC2, CP, SAA4. and COL18A1 are the selected descriptors responsible for interpreting the cell viability values rather than the cellular uptake, which is more related to APOF. Moreover, regarding to cell viability, IGHL8, IGLC2. and CP governed the HeLa cell interaction, while SAA4 and COL18A1 seemed to correlate better to PC3 cell interactions. From another point of view, considering and comparing the number of times that $\mathrm{ZP}$ 

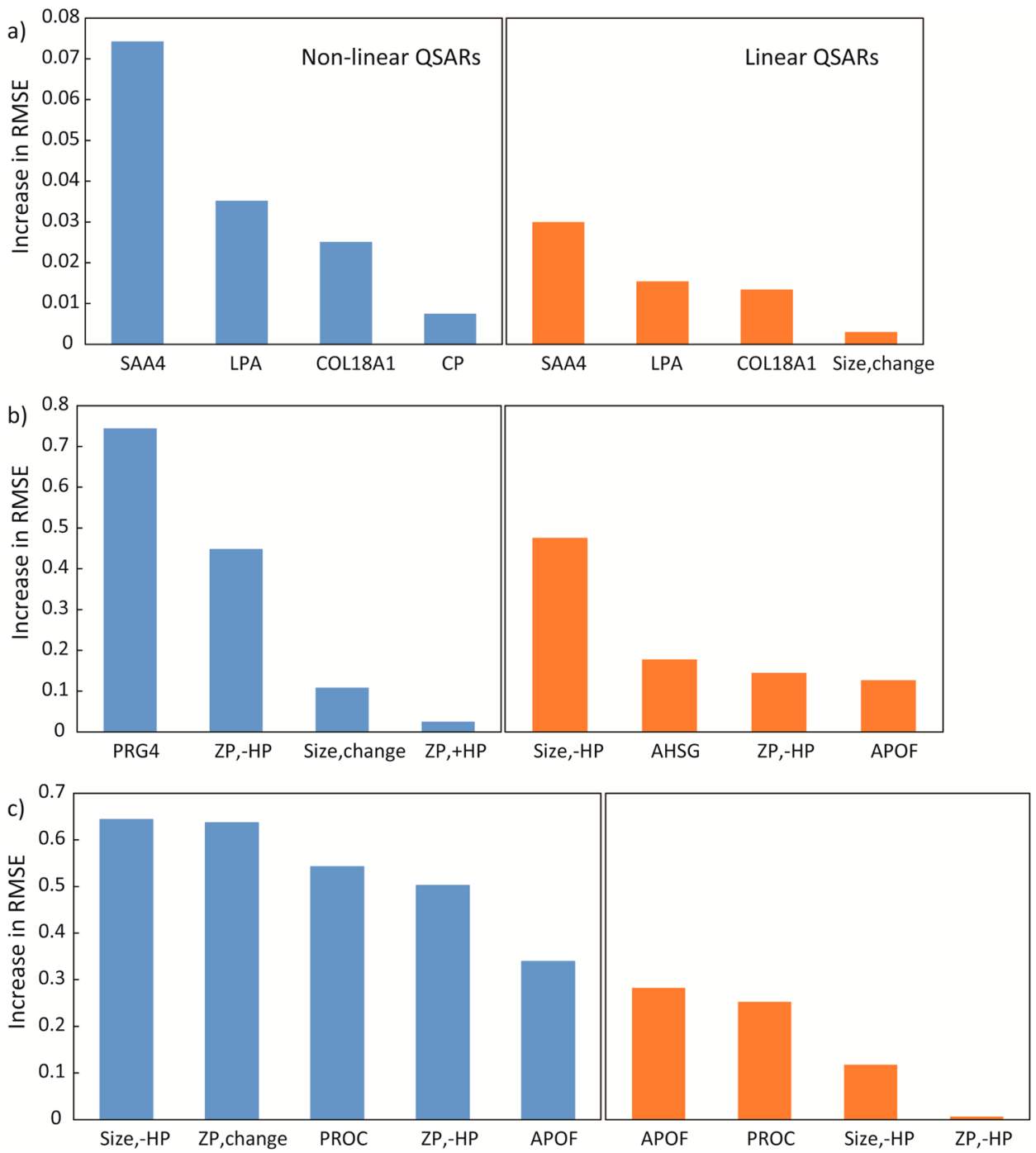

Figure 5. Variables' importance plot measured by sensitivity analysis. Increase in prediction error (RMSE) upon randomly permutation of each descriptor identifies the greater effect of that descriptor in developing the QSAR model. (a) Cell viability, (b) cellular uptake_Flu, (c) cellular uptake Int. The VIPs in this figure are related to PC3 cell line and +HP. The results for HeLa cells and $-\mathrm{HP} /+\mathrm{HP}$ can be found in Figures S10-ST2.

emerged as the important descriptor in -HP in contrast to $+\mathrm{HP}$ subgroups, we can guess that surface charge is an important parameter associated with the synthetic identity of liposomes rather than their biological identity. These phenomena can be further explained by the "charge level-off" issue occurring for charged particles surrounded by serum/ plasma proteins. Moreover, it seemed that zeta potential did not affect the interaction between liposomes and PC3 cells, regarding to the cell viability. In contrast, the appearance of $\mathrm{ZP}$ related descriptors among the important variables for HeLa cells expressed the impact of charge in this cell line. Several other conclusions could be made by a deep screening of the data reported in Table 4. Generally, a consistency was found among the selected variables in linear and nonlinear models, which increased the confidence regarding the significance of the selected descriptors. This consistency is simply justified from the variables frequency plot (Figures 4 and S7-S9).

The most significant variables in each model were ranked by sensitivity analysis (Figures 5 and S10-S12), in which the most important variable caused a higher increase in the prediction error of the resulting model (RMSE) upon its permutation, indicating its significant role for building the model. To make sure that the developed models were significant (i.e., not chance correlations), Y-scrambling test was also performed. The very low $R^{2}$ values after scrambling the $y$ vector in each case indicates the robustness of the corresponding QSAR models (Table 3). Finally, a histogram of the correlation accuracies through the sampling iterations (see Methods) is illustrated in Figure 6 which shows acceptable distribution over the mean $R^{2}$ values reported for each model.

\section{CONCLUSION}

Protein corona fingerprints and physicochemical properties of 17 liposomal formulations were provided to predict multiple biological interactions. The cellular uptake and cell viability of PC3 and HeLa cells were linearly and nonlinearly correlated to the synthetic identity, biological identity, and protein corona of liposomes. Accurate QSAR models were established and the selected descriptors were further used for a thorough investigation on the related nano-bio interactions. Though the significant role of PC formation on nanoparticles fate and behavior has been revealed before in previous studies, this 

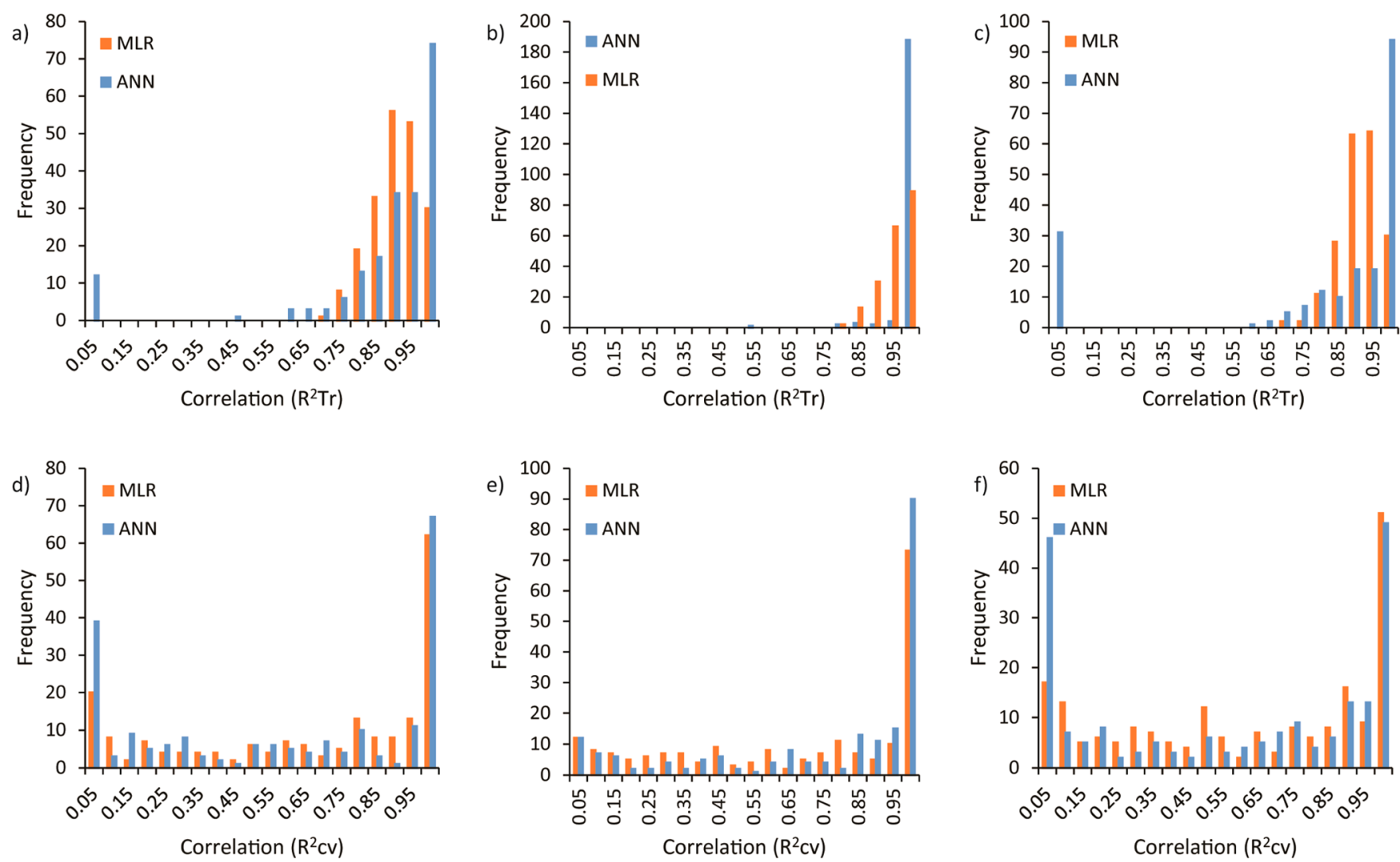

Figure 6. Histograms showing the correlation coefficients obtained from 200 repetitions of modeling on randomly prepared training $\left(R^{2}{ }_{T r}\right)$ and prediction $\left(R_{c v}^{2}\right)$ sets. (a and d) Cell viability; (b and e) cellular uptake_Fluorescence; (c and f) cellular uptake_Intensity. The histograms herein are for PC3 cells and +HP. The results for HeLa cells and $-\mathrm{HP} /+\mathrm{HP}$ are in Figures S13-S15. Please note that $R^{2}{ }_{\text {Tr }}$ is another nomination for $R_{\mathrm{Fit}}^{2}$.

important aspect has not been thoroughly addressed in a "quantitative" framework. Thus, it is essential to take into account different properties of PC (e.g., composition, arrangement, structure, affinity, thickness, density, and conformation) as true evidence of the biological identity of nanoparticles in a quantitative manner and try to disclose what really happens at the nano-bio interfaces. Introducing PC descriptors in this data mining enables researchers to accurately understand and control the interactions of nanoparticles at the nano-bio interfaces which is required for the efficient design of nanoparticles for various medical applications. The use of several cellular responses herein highlighted the fact that different descriptors seem to be relevant to diverse biological processes. The well-established QSARs on different biological end points supplied clues for better understanding and uncovering fundamental mechanisms beyond different liposome-cell interactions.

Identification of specific proteins involved in the related bioresponse produces vital information, offering the potential to be used for improved liposomal design, controlled targeted delivery and other therapeutic goals in which control over nano-bio interactions is highly desired. For instance, the framework presented herein can facilitate the identification of potential inhibitors of well-known targets, such as JQ1 for BRD4 or rhodium complexes as effective STAT3 inhibitors with potent antitumor activity. ${ }^{25,26}$

\section{METHODS}

Samples Preparation. Cationic lipids 1,2-dioleoyl-3-trimethylammonium-propane (DOTAP) and ( $3 \beta-\left[N-\left(N^{\prime}, N^{\prime}-\right.\right.$ dimethylaminoethane)carbamoyl])cholesterol (DC-Chol), zwitterionic lipids dioleoylphosphocholine (DOPC), dioleoylphosphatidylethanolamine (DOPE), DOPE-polyethylene glycol (PEG)-1K, DOPEPEG-2K, DOPE-PEG-5K, 1,2-distearoyl-sn-glycero-3-phosphocholine (DSPC), 1,2-dipalmitoyl-sn-glycero-3-phosphocholine (DPPC), 1,2diarachidoyl-sn-glycero-3-phosphocholine (20:0 PC) and anionic lipids 1,2-dioleoyl-sn-glycero-3-phospho-(1'-rac-glycerol) (DOPG), and 1,2dioleoyl-sn-glycero-3-phosphate (DOPA) were purchased from Avanti Polar Lipids (Alabaster, AL). Sphingosine, Cholesterol (Chol) and DOPE labeled with 7-nitrobenzofurazan (NBD) were purchased from Sigma-Aldrich (St. Louis, MO, USA). All lipids were used without further refinement. Seventeen liposomal formulations were prepared at desired molar ratios between lipid species fixing the molar ratio $\phi=$ neutral lipid/total lipid $(\mathrm{mol} / \mathrm{mol})=0.5$. In the following text, liposomal formulations will be indicated as L1-L17. Each mixture was dissolved in chloroform and the solvent was evaporated under a vacuum for at least $24 \mathrm{~h}$. Lipid films were hydrated to obtain a final lipid concentration of $1 \mathrm{mg} / \mathrm{mL}$ with ultrapure water for size, zeta potential, cytotoxicity, and flow cytometry experiments. For proteomics experiments, lipid films were hydrated with a dissolving buffer (Tris-HCl, pH 7.4, $10 \mathrm{mmol} \mathrm{L}^{-1}$; NaCl, $150 \mathrm{mmol} \mathrm{L}^{-1}$; EDTA, $1 \mathrm{mmol} \mathrm{L}^{-1}$ ) and stored at $4{ }^{\circ} \mathrm{C}$. The obtained liposome solutions were extruded 20 times through a $0.1 \mu \mathrm{m}$ polycarbonate carbonate filter with the Avanti Mini-Extruder (Avanti Polar Lipids, Alabaster, $\mathrm{AL})$.

Size and Zeta Potential. All size and zeta potential measurements were made at $25{ }^{\circ} \mathrm{C}$ on a Zetasizer Nano ZS90 spectrometer (Malvern, U.K.) equipped with a $5 \mathrm{~mW} \mathrm{HeNe}$ laser (wavelength $\lambda=632.8 \mathrm{~nm}$ ) and a digital logarithmic correlator. The normalized intensity 
autocorrelation functions were analyzed using the CONTIN method, ${ }^{27}$ to obtain the distribution of the diffusion coefficient $D$ of the particles. This coefficient is converted into an effective hydrodynamic radius $R_{\mathrm{H}}$ using the Stokes-Einstein equation $R_{\mathrm{H}}=K_{\mathrm{B}} T$ / $(6 \pi \eta D)$, where $K_{\mathrm{B}} T$ is the thermal energy and $\eta$ is the solvent viscosity. The electrophoretic mobility measurements were performed by means of the laser Doppler electrophoresis technique, by the same apparatus used for size measurements. The mobility $u$ was converted into the zeta potential using the Smoluchowski relation, zeta potential $=u \eta / \varepsilon$, where $\eta$ and $\varepsilon$ are, respectively, the viscosity and the permittivity of the solvent phase. A volume of $100 \mu \mathrm{L}$ of each liposome formulation was mixed with an equal amount of HP and the mixture was incubated for $1 \mathrm{~h}$ at $37^{\circ} \mathrm{C}$. After the incubation in HP, the samples were centrifuged at $14000 \mathrm{rpm}$ for $15 \mathrm{~min}$ at $4{ }^{\circ} \mathrm{C}$ to pellet the particle-protein complexes and separate them from the supernatant. The pellet was then resuspended in $1 \mathrm{~mL}$ of PBS and centrifuged again for $3 \mathrm{~min}$ at $14000 \mathrm{rpm}$ at $4{ }^{\circ} \mathrm{C}$. Three washing steps were made before resuspension of the final pellet. Size and zeta potential results are given as mean \pm standard deviation of five replicates.

Cell Lines. Human prostate cancer cell line (PC3), derived from human bone prostate cancer metastasis, was purchased from ATCC (Manassas, VA, USA). PC3 cells were maintained in RPMI 1640 medium supplemented with $2 \mathrm{mM}$ L-glutamine, $100 \mathrm{IU} / \mathrm{mL}$ penicillin/ streptomycin, $1 \mathrm{mM}$ sodium pyruvate, $10 \mathrm{mM}$ HEPES, $1.5 \mathrm{mg} / \mathrm{L}$ sodium bicarbonate, and $10 \%$ fetal bovine serum (FBS) (SigmaAldrich, St. Louis, MO, USA). Human cervical cancer cell line (HeLa), derived from human cervix adenocarcinoma, was purchase from ATCC (Manassas, VA, USA). HeLa cells were maintained in Eagle's Minimum Essential Medium (EMEM) supplemented with $2 \mathrm{mM} \mathrm{L-}$ glutamine, $100 \mathrm{IU} / \mathrm{mL}$ penicillin/streptomycin, $1 \mathrm{mM}$ sodium pyruvate, $10 \mathrm{mM}$ HEPES, $1.5 \mathrm{mg} / \mathrm{L}$ sodium bicarbonate, and $10 \%$ fetal bovine serum (FBS).

Protein Assay. To quantify the amount of adsorbed proteins on liposomes after incubation with HP, we used the Protein Assay reagent (Pierce, Thermo Scientific, Waltham, MA, USA), according to manufacturer's protocol. Briefly, each liposome sample was incubated with $\mathrm{HP}$ for $1 \mathrm{~h}$ at $37{ }^{\circ} \mathrm{C}$. Then, liposome-HP complexes were centrifuged at $14000 \mathrm{rpm}$ for $15 \mathrm{~min}$ at $4{ }^{\circ} \mathrm{C}$ and washed 3 times with PBS, and the resulting pellet was resuspended in urea $8 \mathrm{~mol} / \mathrm{L}$ $\mathrm{NH}_{4} \mathrm{CO}_{3} 50 \mathrm{mmol} / \mathrm{L}$. Ten microliters of each sample was placed into a 96-multiwell plate and then $50 \mu \mathrm{L}$ of Protein Assay reagent was added. The measures were performed in triplicate. The multiwell was covered, mixed on a plate shaker, and incubated at room temperature for $5 \mathrm{~min}$. The absorbance of each sample, standard and blank was measured with the Glomax Discover System (Promega, Madison, WI, USA) at $660 \mathrm{~nm}$. The protein concentration was calculated using the standard curve.

Protein Identification and Quantification. A total of $100 \mu \mathrm{L}$ of liposomes $(1 \mathrm{mg} / \mathrm{mL})$ was incubated with an equal volume of human plasma at $37{ }^{\circ} \mathrm{C}$. Following 1-h incubation, liposome-protein complexes were centrifuged ( $15 \mathrm{~min}$ at $14000 \mathrm{rpm}$ ) to remove loosely bound proteins (i.e., the soft corona). Then, pellets were accurately washed three times with $100 \mu \mathrm{L}$ of the dissolving buffer. Protein denaturation, digestion, and desalting were performed by a robust methodology that is commonly followed to separate liposomeprotein complexes from unbound proteins. ${ }^{11}$ The following step was sample lyophilization. This was achieved by means of a Speed-Vac apparatus (mod. SC 250 Express; Thermo Savant, Holbrook, NY, USA). Subsequently, samples were reconstituted with $0.1 \% \mathrm{HCOOH}$ solution (final concentration $0.32 \mathrm{mg} / \mathrm{mL}$ ) and stored at $80{ }^{\circ} \mathrm{C}$ until nano liquid chromatography (LC) tandem mass spectrometry (MS/ MS) analysis. Tryptic peptides were examined by a dedicated nano-LC system (Dionex Ultimate 3000, Sunnyvale, CA, USA) connected to a hybrid mass spectrometer (Thermo Fisher Scientific Bremen, Germany), equipped with a nanoelectrospray ion source. Comprehensive experimental details can be found elsewhere. ${ }^{11}$ Data analysis and protein validation were made according to standard procedures. Briefly, Xcalibur (v.2.07, ThermoFisher Scientific) raw data files were submitted to Proteome Discover (1.2 version, Thermo Scientific) for database search using Mascot (version 2.3.2 Matrix Science). Data were searched against SwissProt database (v 57.15, 20266 sequences) using the decoy search option of Mascot. Final protein quantification analysis was made by Scaffold software that allows the normalization of the spectral countings (normalized spectral countings, NSCs). For each identified protein, the mean value of NSCs was normalized to the protein molecular weight (MWNSC) to obtain the relative protein abundance. Statistical significance of data was guaranteed by replicating the procedure for nine samples. Data of relative protein abundance were provided as mean \pm standard deviation.

Analysis of Cell Viability. To investigate the potential toxicity arising from bare NPs, cell viability of prostate cancer cells PC3 and cervical cancer cells HeLa was assessed by 3-(4,5-dymethyl thiazol 2y1)-2,5-diphenyl tetrazolium bromide (MTT, mitochondrial respiration analysis; Sigma-Aldrich), according to Mosmann protocol. Briefly, PC 3 and HeLa cells were seeded on 96-wells plate (10 000 cells/well). The day after, cells were treated with $10 \mu \mathrm{g} / \mathrm{mL}$ of each formulation in Optimem medium (Life Technologies, Carlsbad, CA) for $24 \mathrm{~h}$. Then, MTT was added to each well at the final concentration of $0.5 \mathrm{mg} / \mathrm{mL}$, and after $4 \mathrm{~h}$ of incubation at $37^{\circ} \mathrm{C}$, the formazan salt was dissolved with $100 \mu \mathrm{L}$ of isopropylic alcohol. The absorbance of each well was measured with Glomax Discover System (Promega, Madison, WI, USA), a ready-to-use high-performance multimode detection instrument. The viability was calculated for each treatment as (OD of treated cells/OD of control cells) $\times 100$.

Flow Cytometry. To investigate cellular uptake of nanoparticles in PC3 and HeLa cell lines, each of the 17 liposomal formulations was synthesized with the addition of DOPE-NBD. In all the formulations, the concentration of fluorescently labeled NBD-DOPE was $7 \times 10^{-3}$ $\mathrm{mg} / \mathrm{mL}$ (fluorescent lipid/total lipid molar ratio $=5 / 1000$ ). Bare liposomes and liposome-HP complexes were administered to cells cultured with serum-free medium. PC3 and HeLa cells were plated at 200000 cells $/ \mathrm{mL}$ in 12-well dishes. After $24 \mathrm{~h}$, cells were incubated for $3 \mathrm{~h}$ with $10 \mu \mathrm{g} / \mathrm{mL}$ of NBD-labeled liposomes. After the treatment, cells were detached with trypsin/ethylenediaminetetraacetic acid (EDTA), washed twice with cold PBS, and run on a BD LSFORTESSA (BD Biosciences, San Jose, CA, USA). Cells were gated using forward vs side scatter to exclude debris. Data were analyzed using FlowJo software (FlowJo LLC data analysis software, Ashland, OR, USA).

Descriptor Generation. A set of 656 descriptors was collected for 17 liposome formulations, consisting of 633 PC fingerprints (PCF) and 23 liposome physicochemical properties (LPP). The full descriptions of all the 656 descriptors together with their values are given in Table S1 of Supporting Information. The first action taken on the data set was initial screening for removing highly correlated descriptors. Descriptors with zero or constant values and also the ones with correlation coefficients higher than $80 \%$ with each other (multicollinear) were removed from the initial pool of variables. Thus, the data set consisting of 17 liposome formulations and 148 descriptors was prepared for the QSAR studies. Table 2 represents the two classes of descriptors used in the QSAR analysis.

QSAR Modeling. Linear and nonlinear QSAR models were used to predict 12 different sets of biological end points for 17 liposome formulations. The response vectors were $\log _{2}$ transformed and included cell viability, cellular uptake fluorescent, and cellular uptake_intensity, each measured for two cell types (PC3 and HeLa) and with/without HP incubation $(=3 \times 2 \times 2)$.

In Multiple Linear Regression (MLR), ${ }^{28,29}$ a simple linear regression was used to fit the data and minimize the root-meansquare error between the measured $\left(y_{i}\right)$ and predicted $\left(\hat{y}_{i}\right)$ responses for each liposome:

$$
\mathrm{RMSE}=\sqrt{\frac{1}{n} \sum_{i=1}^{n}\left(y_{i}-\hat{y_{i}}\right)^{2}}
$$

Another parameter that is widely used for evaluating the performance of a QSAR model is the coefficient of determination: 


$$
R^{2}=1-\sum_{i=1}^{n}\left(y_{i}-\hat{y}_{i}\right)^{2} / \sum_{i=1}^{n}\left(y_{i}-\bar{y}_{i}\right)^{2}
$$

$\bar{y}_{i}$ is the mean response measured for each liposome. Depending on the $n$ samples coming from the training set or the prediction set, for which $\hat{y}_{i}$ is calculated, the coefficient of determination would be referred to as $R_{\mathrm{fit}}^{2}$ or $R_{\mathrm{cv}}^{2}$, respectively.

As a nonlinear model, Artificial Neural Networks (ANN) was utilized to correlate the LPP and PCF descriptors to different bioresponse profiles. ANN with a layered structure is a mathematical system stimulating the biological neural network, consisting of neurons and synapses. The measured variables are presented to an input layer and are processed, by one or more intermediate ("hidden") layers, to produce one or more outputs. Synapses are responsible for the connections between these input, hidden and output neurons. The more the strength of the synapses, the heavier the weight of the connections between different neuron layers. The weights and biases are two internal parameters in a neural network that iteratively change during training in order to minimize a performance function which is usually the discrepancy between the observed and predicted values (i.e., error between network inputs and target outputs). The performance function herein was set to the root minimum square error, as shown in the above formula. Hence, the neural network will be trained until the desired degree of accuracy is achieved. There are several algorithms for training multilayer feed forward neural networks.

In this study, we used Bayesian Regularization $(B R)^{30}$ in combination to Levenberg-Marquardt training algorithm to develop nonlinear ANN models. BR is a powerful optimization procedure in ANN modeling and is a beneficial approach to deal with the overfitting (i.e., occurs when the model has memorized the training samples but has not learned to generalize to new samples, leading to too high degree of accuracy for training samples but poor prediction for new samples). BR as a solution to this regularization problem modifies the performance function so that the network will have smaller weights and biases and its response will be smoother and less likely to overfit. Unlike most of the linear models, ANN does not start by assuming a particular type of mathematical relationship between the input and output variables. This makes ANN particularly useful when the underlying model is unknown. However, the operation of ANN is like a black box, comprising complicated mathematical and systematic relations. Therefore, in QSAR models, ANNs are generally used as purely predictive tools rather than as an aid in understanding structure-property trends, although great prediction ability, high reproducibility, and generalization power of BRANN modeling makes it superior and offers the opportunity to explore nonlinear phenomena and curved manifold. Since biological subjects may have nonlinear characteristics, ANN techniques are beneficial for discovering the possible relationship between the input descriptors and the output bioresponses.

For each liposome, the biological responses where predicted using both MLR and ANN models. The QSAR analysis began by randomly splitting the data set into two subsets, called prediction set (20\% of the raw data) and training set (the remaining $80 \%$ of the raw data). The training set is used to build the model which is then validated by the prediction set. To achieve a good QSAR model, a minimal set of information-rich descriptors is needed, requiring a suitable variable selection step to be applied. ${ }^{31}$ Stepwise regression as a simple yet powerful variable selection method is an iterative selection procedure which starts from a variable with the largest empirical correlation with the dependent variable (response). Each iteration of stepwise regression includes two phases: the inclusion phase in which each remaining variable is subjected to a partial $F$-test, and the variable with the $F$-value larger than a critical ' $F$-to-enter' value is inserted in the model; and the exclusion phase, in which the variable with the $F$-value smaller than a critical ' $F$-to-remove' value will be removed from the model and returned to the pool of variables still available for the selection. Thus, in each iteration of the initial splitting, the training set was applied to stepwise regression variable selection method to choose the best descriptors among the large number of descriptors.
To deal with overfitting in both variable selection and modeling steps, Monte Carlo cross validation was used. ${ }^{32-34}$ This technique involves a large number of random splits of the data set repeatedly, in each of which the available data is divided into two groups to be used for the training and testing. The criterion (e.g., root-mean-square error or coefficient of determination) is averaged over all the repeated splits, so as to not tie the measure to one particular division of the data and to be representative of the whole data. For this reason, the training set was itself randomly divided into two subsets (train and test) for 100 times and the most frequent variables were chosen afterward. In each iteration of the outer loop (200 runs), after 100 times of repeating variable selection, the prediction set with the best selected descriptors was then applied to the linear MLR or nonlinear ANN model to assess its prediction ability. The algorithm followed this procedure until 200 runs of the outer loop were completed. The results were averaged over the corresponding repetitions. It should be noted that the selected descriptors from each iteration were recorded and the best ones where subsequently chosen based on the variables frequency plot which was illustrated after completing the whole iterations. The descriptors with the highest frequency having reasonable difference with others were represented as the best of the best selected descriptors, most suitable for correlation.

Sensitivity Analysis and Descriptor Importance. To assess the importance of the selected descriptors (which were identified based on their higher frequency in incorporating in the model development), sensitivity analysis was used to measure their relative importance in the predictive ability of the model. ${ }^{35}$ In this approach, each descriptor was in turn randomly scrambled and the RMSE of the resulting model was calculated and compared with that when all the descriptors were available. Therefore, an increase in RMSE (or decrease in the model performance) according to scrambling a selected descriptor represents the relative importance of the corresponding descriptor. In other words, the importance priority of the selected descriptors is achieved based on the amount of decrease in the model performance. In addition, the frequency plot of descriptors illustrates how many times each descriptor has participated in the QSAR procedure. The higher a variables' frequency, the more it has been displayed in variable selection and the better it correlates to activities.

Model Robustness. In addition to Monte Carlo cross validation which was used to quantify the prediction abilities of the developed QSAR models (the histogram of Monte Carlo cross validation correlation coefficients shows the distribution of $R_{\mathrm{cv}}^{2}$ in 200 repetitions), the robustness of the models was also further examined. The statistical significance of QSAR models was checked by comparing its measure of fit to the average measure of fit when the response vector is completely randomized. ${ }^{25,26}$ This approach is called Yrandomization or Y-scrambling, and is used to check if the model is built upon chance correlations. By scrambling the response vector, it is expected to get much lower $R^{2}$ values, indicating the significance of the original QSAR model which has been now destroyed by this shuffling. Thus, a 100-round Y-randomization was applied to the data to confirm that the developed models were not chance correlations.

Applicability Domain. William's graph as the most common method was applied to define the applicability domain of each QSAR model. $^{34,36}$ The applicability domain represents the theoretical descriptor space in which reliable QSAR predictions can be made. William's graph depicts a QSAR's applicability domain with a twodimensional scatter plot. The first dimension which reflects the structural similarity between one sample and all other samples in the training set is the "leverage" which is calculated for each sample $\left(x_{i}\right)$ in the data set $(X)$ by

$$
h_{i}=x_{i}\left(X^{\mathrm{T}} X\right)^{-1} x_{i}^{\mathrm{T}}
$$

The leverage quantitatively expresses the distance of a given liposome to the center of all liposome samples used for QSAR development in the descriptor space. Liposomes with smaller $h$ value are more similar to the data set used for QSAR modeling, meaning that they are within the descriptor space in which the bioresponse can be predicted more 
reliably. A threshold leverage value, which covers about 99\% of normally distributed training samples, is usually calculated by

$$
h^{*}=\frac{3(m+1)}{n}
$$

where $m$ and $n$ are the number of descriptors in the model and number of training samples, respectively. Liposomes with leverage values higher than this warning threshold value are defined as outliers, meaning that they do not fit well in the current model, as others.

The second dimension of William's graph is the standardized prediction error:

$$
\varepsilon_{i}=\frac{e_{i}-\bar{e}}{\sigma_{\mathrm{e}}}
$$

where $\bar{e}$ and $\sigma_{\mathrm{e}}$ are the average and standard deviation of the residual error $\left(=y_{i}-\hat{y}_{i}\right)$, respectively. A sample is considered as outlier if its absolute $\varepsilon_{i}$ value is greater than 3 .

\section{AUTHOR INFORMATION}

\section{Corresponding Authors}

*E-mail: giulio.caracciolo@uniroma1.it.

*E-mail: mahmoudi-m@tums.ac.ir, Mahmoudi@stanford.edu.

Notes

The authors declare no competing financial interest.

\section{ACKNOWLEDGMENTS}

M.M. would like to thank Tehran University of Medical Sciences for supporting this work (Grant No. 94-04-33-31032). G.C. and D.P. acknowledge support by the Italian Minister for University and Research (MIUR) (Futuro in Ricerca 2008, Grant No. RBFR08TLPO).

\section{REFERENCES}

(1) Allen, T. M.; Cullis, P. R. Liposomal Drug Delivery Systems: From Concept to Clinical Applications. Adv. Drug Delivery Rev. 2013, $65,36-48$

(2) Lasic, D. D.; Papahadjopoulos, D. Medical Applications of Liposomes; Elsevier Science: Amsterdam, 1998; pp 1-13.

(3) Al-Jamal, W.; Kostarelos, K. Liposomes: From a Clinically Established Drug Delivery System to a Nanoparticle Platform for Theranostic Nanomedicine. Acc. Chem. Res. 2011, 44, 1094-1104.

(4) Lynch, I.; Salvati, A.; Dawson, K. A. Protein-nanoparticle Interactions: What Does the Cell See? Nat. Nanotechnol. 2009, 4, 546-547.

(5) Walczyk, D.; Bombelli, F. B.; Monopoli, M. P.; Lynch, I.; Dawson, K. A. What the Cell "sees" in Bionanoscience. J. Am. Chem. Soc. 2010, 132, 5761-5768.

(6) Lundqvist, M.; Stigler, J.; Elia, G.; Lynch, I.; Cedervall, T.; Dawson, K. A. Nanoparticle Size and Surface Properties Determine the Protein Corona with Possible Implications for Biological Impacts. Proc. Natl. Acad. Sci. U. S. A. 2008, 105, 14265-14270.

(7) Walkey, C. D.; Chan, W. C. W. Understanding and Controlling the Interaction of Nanomaterials with Proteins in a Physiological Environment. Chem. Soc. Rev. 2012, 41, 2780-2799.

(8) Caracciolo, G. Liposome-Protein Corona in a Physiological Environment: Challenges and Opportunities for Targeted Delivery of Nanomedicines. Nanomedicine 2015, 11, 543-557.
(9) Pozzi, D.; Colapicchioni, V.; Caracciolo, G.; Piovesana, S.; Capriotti, A. L.; Palchetti, S.; Grossi, S. D.; Riccioli, A.; Amenitsch, H.; Lagana, A. Effect of Polyethyleneglycol (PEG) Chain Length on the Bio-nano-interactions Between PEGylated Lipid Nanoparticles and Biological Fluids: From Nanostructure to Uptake in Cancer Cells. Nanoscale 2014, 6, 2782-2792.

(10) Capriotti, A. L.; Caracciolo, G.; Cavaliere, C.; Foglia, P.; Pozzi, D.; Samperi, R.; Lagana, A. Do Plasma Proteins Distinguish Between Liposomes of Varying Charge Density? J. Proteomics 2012, 75, 19241932.

(11) Barran Berdon, A. L.; Pozzi, D.; Caracciolo, G.; Capriotti, A. L.; Caruso, G.; Cavaliere, C.; Riccioli, A.; Palchetti, S.; Lagana, A. Time Evolution of Nanoparticle-Protein Corona in Human Plasma: Relevance for Targeted Drug Delivery. Langmuir 2013, 29, 64856494.

(12) Lundqvist, M. Nanoparticles: Tracking Protein Corona over Time. Nat. Nanotechnol. 2013, 8, 701-702.

(13) Mahmoudi, M.; Abdelmonem, A. M.; Behzadi, S.; Clement, J. H.; Dutz, S.; Ejtehadi, M. R.; Hartmann, R.; Kantner, K.; Linne, U.; Maffre, P.; Metzler, S.; Moghadam, M. K.; Pfeiffer, C.; Rezaei, M.; Ruiz-Lozano, P.; Serpooshan, V.; Shokrgozar, M. A.; Nienhaus, G.; Ulrich; Parak, W. J. Temperature: The "Ignored" Factor at the NanoBio Interface. ACS Nano 2013, 7, 6555-6562.

(14) Caracciolo, G.; Pozzi, D.; Capriotti, A. L.; Cavaliere, C.; Foglia, P.; Amenitsch, H.; Lagana, A. Evolution of the Protein Corona of Lipid Gene Vectors as a Function of Plasma Concentration. Langmuir 2011 27, 15048-15053.

(15) Hajipour, M. J.; Laurent, S.; Aghaie, A.; Rezaee, F.; Mahmoudi, M. Personalized Protein Coronas: A "key" Factor at the Nanobiointerface. Biomater. Sci. 2014, 2, 1210-1221.

(16) Caracciolo, G.; Pozzi, D.; Capriotti, A. L.; Cavaliere, C.; Piovesana, S.; La Barbera, G.; Amici, A.; Lagana, A. The LiposomeProtein Corona in Mice and Humans and Its Implications for in vivo Delivery. J. Mater. Chem. B 2014, 2, 7419-7428.

(17) Walkey, C. D.; Olsen, J. B.; Song, F.; Liu, R.; Guo, H.; Olsen, D. W. H.; Cohen, Y.; Emili, A.; Chan, W. C. W. Protein Corona Fingerprinting Predicts the Cellular Interaction of Gold and Silver Nanoparticles. ACS Nano 2014, 8, 2439-2455.

(18) Liu, K.; Jiang, W.; Walkey, C. D.; Chan, W. C. W.; Cohen, Y. Prediction of Nanoparticles-Cell association based on Corona Proteins and Physicochemical Properties. Nanoscale 2015, 7, 9664-9675.

(19) Pozzi, D.; Caracciolo, G.; Digiacomo, L.; Colapicchioni, V.; Palchetti, S.; Capriotti, A. L.; Cavaliere, C.; Zenezini Chiozzi, R.; Puglisi, A.; Lagana, A. The biomolecular corona of nanoparticles in circulating biological media. Nanoscale 2015, 7, 13958-13966.

(20) Tenzer, S.; Docter, D.; Kuharev, J.; Musyanovych, A.; Fetz, V.; Hecht, R.; Schlenk, F.; Fischer, D.; Kiouptsi, L.; Reinhardt, C.; Landfester, K.; Schild, H.; Maskos, M.; Knauer, S. K.; Stauber, R. H. Rapid Formation of Plasma Protein Corona Critically Affects Nanoparticle Pathophysiology. Nat. Nanotechnol. 2013, 8, 772-781.

(21) Doorley, G. W.; Payne, C. K. Cellular Binding of Nanoparticles in the Presence of Serum Proteins. Chem. Commun. 2011, 47, 466468 .

(22) Huhn, D.; Kantner, K.; Geidel, C.; Brandholt, S.; Cock, I. D.; Soenen, S. J. H.; Rivera Gil, P.; Montenegro, J. M.; Braeckmans, K.; Mullen, K.; Nienhaus, G. U.; Klapper, M.; Parak, W. J. PolymerCoated Nanoparticles Interacting with Proteins and Cells: Focusing on the Sign of the Net Charge. ACS Nano 2013, 7, 3253-3263.

(23) Lynch, I.; Dawson, K. A. Protein-Nanoparticle Interactions. Nano Today 2008, 3, 40-47.

(24) Tenzer, S.; Docter, D.; Rosfa, S.; Wlodarski, A.; Kuharev, J.; Rekik, A.; Knauer, S. K.; Bantz, C.; Nawroth, T.; Bier, C.; Sirirattanapan, J.; Wolf, M.; Lennart, T.; Reinhard, Z.; Michael, M.; Hansjorg, S.; Stauber, R. H. Nanoparticle Size Is a Critical Physicochemical Determinant of the Human Blood Plasma Corona: A Comprehensive Quantitative Proteomic Analysis. ACS Nano 2011, 5, 7155-7167.

(25) Filippakopoulos, P.; Qi, J.; Picaud, S.; Shen, Y.; Smith, W. B.; Fedorov, O.; Morse, E. M.; Keates, T.; Hickman, T. T.; Felletar, I.; 
Phipott, M.; Munro, S.; McKeown, M. R.; Wang, Y.; Christie, A. L.; West, N.; Cameron, M. J.; Schwartz, B.; Heightman, T. D.; Thangue, N. L.; French, C. A.; Wiest, O.; Kung, A. L.; Knapp, S.; Bradner, J. E. Selective Inhibition of BET Bromodomains. Nature 2010, 468, 10671073.

(26) Ma, D. L.; Liu, L. J.; Leung, K. H.; Chen, Y. T.; Zhong, H. J.; Chan, D. S. H.; Wang, H. M. D.; Leung, C. H. Antagonizing STAT3 Dimerization with a Rhodium(III) Complex. Angew. Chem., Int. Ed. 2014, 53, 9178-9182.

(27) Provencher, S. W. A Constrained Regularization Method for Inverting Data Represented by Linear Algebraic or Integral Equations. Comput. Phys. Commun. 1982, 27, 213-227.

(28) Gemperline, P. In Practical Guide to Chemometrics, 2nd ed.; CRC Press, Taylor and Francis, LLC, 2006; pp 111-116.

(29) Miller, J. N.; Miller, J. C. In Statistics and Chemometrics for Analytical Chemistry, 5th ed.; Pearson: Harlow, U.K., 2005; pp 228237.

(30) MacKay, D. J. C. A Practical Bayesian Framework for Backpropagation Networks. Neural Comput. 1992, 4, 448-472.

(31) Anderson, C. M.; Bro, R. Variable Selection in Regression. J. Chemom. 2010, 24, 728-737.

(32) Gramatica, P. Principles of QSAR Models Validation: Internal and External. QSAR Comb. Sci. 2007, 26, 694-701.

(33) Eriksson, L.; Jaworska, J.; Worth, A. P.; Cronin, M. T. D.; McDowell, R. M.; Gramatica, P. Methods for Reliability, Uncertainty Assessment, and Applicability Evaluations of Classification and Regression Based QSARs. Environ. Health Perspect. 2003, 111, $1361-1375$

(34) Puzyn, T.; Leszczynska, D.; Leszczynski, J. Toward the Development of 'Nano-QSARs': Advances and Challenges. Small 2009, 5, 2494-2509.

(35) Guha, R.; Jurs, P. C. Interpreting Computational Neural Network QSAR models: A Measure of Descriptor Importance. J. Chem. Inf. Model. 2005, 45, 800-806.

(36) Tropsha, A.; Gramatica, P.; Gombar, P. V. The Importance of Being Earnest: Validation is the Absolute Essential for Successful Application and Interpretation of QSPR Models. QSAR Comb. Sci. 2003, 22, 69-77. 